Graduate A eronautical Laboratories California Institute of $T_{\text {echnology }}$

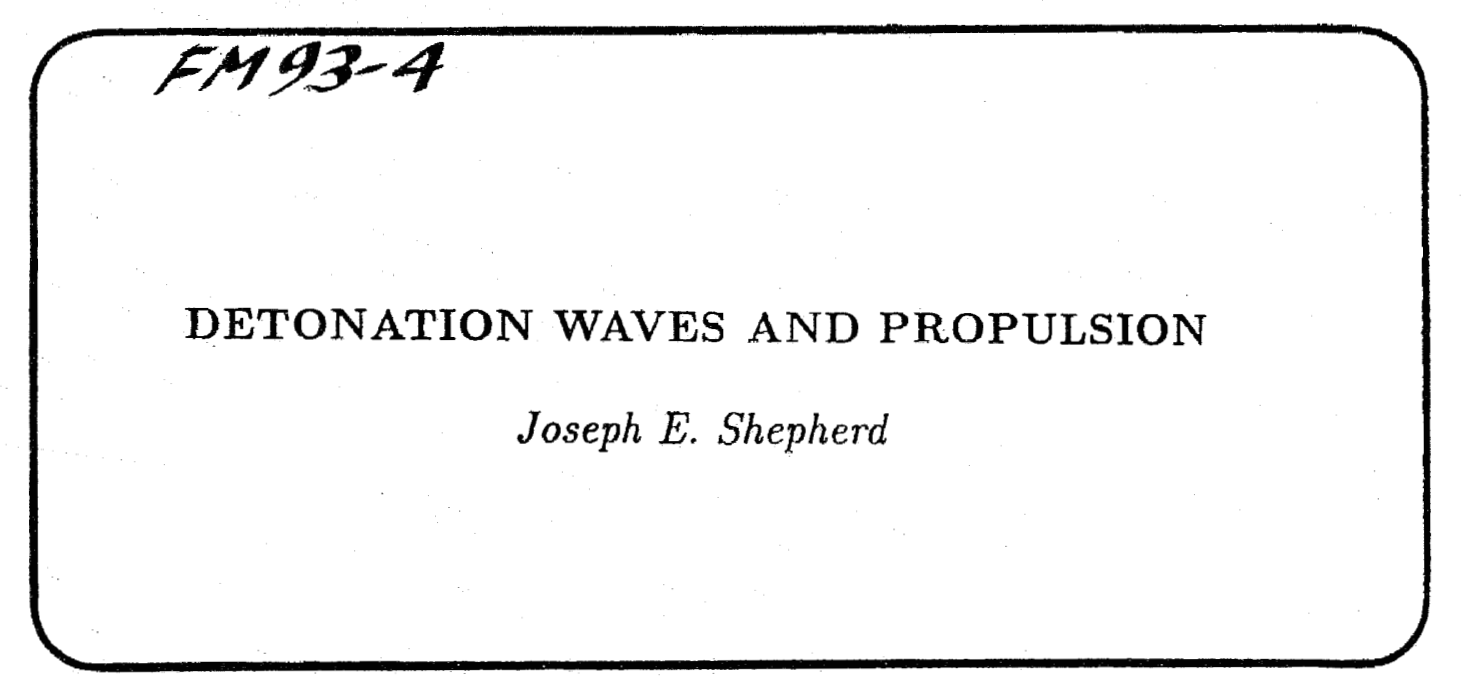

Firestone Flight Sciences Laboratory

Guggenheim Aeronautical Laboratory

Karman Laboratory of Fluid Mechanics and Jet Propulsion 


\title{
DETONATION WAVES AND PROPULSION
}

\author{
Joseph E. Shepherd \\ Graduate Aeronautical Laboratories \\ California Institute of Technology \\ Pasadena, CA 91125
}

\begin{abstract}
The possibility of using a detonation wave as the key combustion system for supersonic propulsion is examined. A brief review of propagating detonations is provided first. This review emphasizes the unique and unstable nature of the coupling between reaction zone and shock waves that characterize detonations. The theory of idealized, steady, oblique detonation waves and their reaction zone structure are summarized. The evidence for the existence of stabilized or steady oblique detonations is discussed. Experiments with multiple layers of explosive and projectiles fired into explosive gases are examined. There are a variety of reasons that these previous studies have failed to produce stabilized detonations. A brief catalog of difficulties is provided and based on analogies with our knowledge of propagating detonations, a set of criteria are proposed for the existence and stability of stabilized detonations. The problems of initiation and instability are examined for the situation of a flow over a wedge.
\end{abstract}

\section{Introduction}

One of the most intriguing possibilities for supersonic propulsion is using a combustor based on a stabilized detonation wave. A number of studies of this concept were made in the 1950s and 1960s (a useful set of historical references are given in Pratt et al. 1991) and recently there has been a renewed interest in this subject. The concept is, in principle, quite simple as shown in Fig. 1. Fuel is mixed thoroughly into a supersonic flow of air within the engine duct and a detonation wave is stabilized by inserting a bend or obstruction into the flow. The thermal energy resulting from the combustion is converted to kinetic energy in the nozzle to produce thrust. Simple engine performance estimates (Dunlap et al. 1958, Sargent and Gross

To be published in Combustion in High-Speed Flows, Eds. Jackson, T., Buckmaster, J., and Kumar, Kluwer, 1994. Presented at the 2nd ICASE/NASA Combustion Workshop, Hampton, VA, October 13, 1992. 
1960, Ostrander et al. 1987 and Atamanchuk and Sislian 1990) and vehicle design studies (Atamanchuk and Sislian 1991) indicate that this concept may have some merit. However, it is speculative and the main focus of present-day high-speed propulsion research (Murthy and Curran 1991) is on the more traditional supersonic combustion ramjet or scramjet combustor approach.

Conceptually, the oblique detonation wave is analogous to an oblique shock wave (Thompson 1972) with chemical reaction. As shown in Fig. 2, the wave extends at an angle $\beta$ into the flow and turns the flow by angle $\theta$. Such a configuration is conceivable as long as the flow upstream is moving faster that the characteristic detonation wave speed, the Chapman-Jouguet (CJ) velocity. Since detonations are supersonic combustion waves that propagate with a relative Mach number between 4 and 10, only a modest amount of compression is required in the inlet diffuser in comparison with the traditional gas-turbine combustor. This is what makes the concept so attractive for propelling supersonic flight.

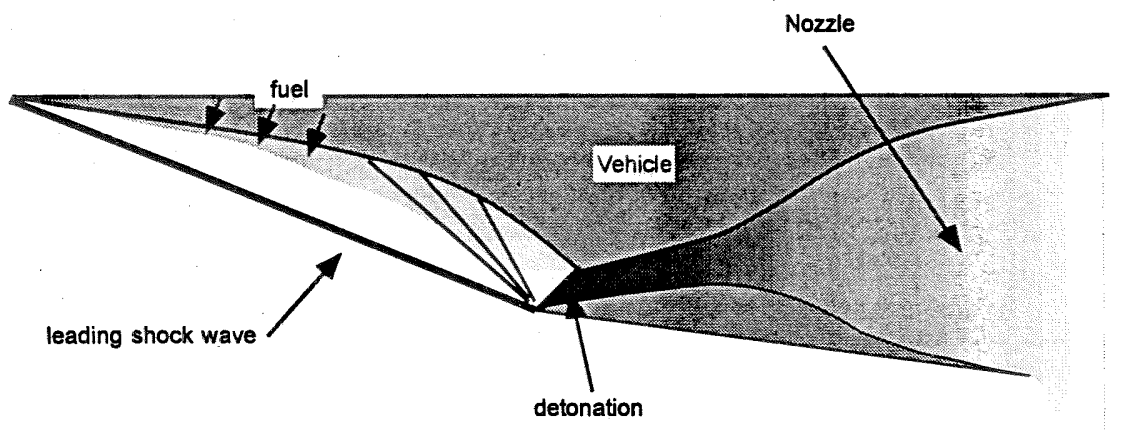

Figure 1: Schematic diagram of a propulsion system based on a standing detonation wave.

Despite the apparent simplicity of this approach, there are a number of practical issues that must be resolved before a detonation wave-based engine can be constructed. Foremost is the issue of detonation stability. Although propagating detonations have been extensively investigated, little is known about the initiation and stabilization of steady detonation waves. For propagating detonations, it is known that a minimum width duct and a minimum initiation 
energy are required. The precise corresponding criteria for steady detonation waves have yet to be defined. One of the aims of this paper is to suggest criteria based on our knowledge of propagating detonations.

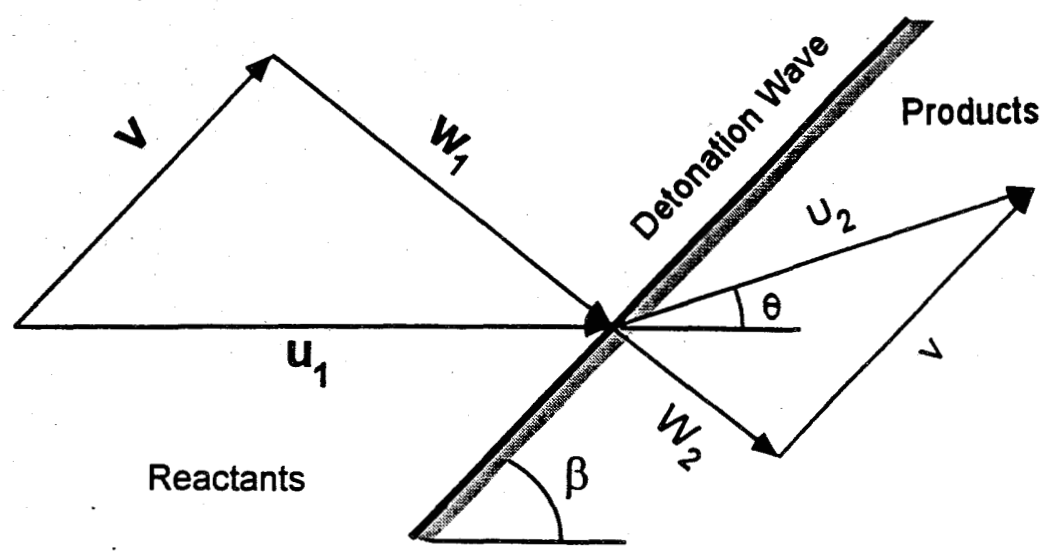

Figure 2: Flow deflection and velocity vectors associated with an idealized oblique detonation wave.

There are many other issues that are crucial to the performance of a detonation wave engine. Key among these are: the adjustment of the duct and inlet geometry as a function of flight Mach number; mixing of fuel with the air while controlling pre-ignition; conversion of gas chemical energy to kinetic energy within the exhaust nozzle (Harradine et al. 1990 and Sangiovanni et al. 1993). It is particularly important to have realistic analyses and reliable experimental data in order to construct performance models and predict the behavior of full-scale engines. This is due to the intrinsically marginal performance of airbreathing propulsion at hypersonic flight speeds. The potential energy addition due to combustion is a much smaller fraction of the stagnation enthalpy of the free-stream flow at hypersonic speeds as compared to traditional low-speed propulsion.

At the present time, it is not clear if the construction of an engine using detonation waves is feasible. A very substantial amount of engineering research and development is needed before this can be decided. Even the most basic issues such as the existence and 
stability of a steady detonation wave inside an engine duct are quite controversial. As we shall see, the experimental evidence is scant and contradictory and the theory is far from definitive. Our knowledge of detonations is almost exclusively based on propagating waves and almost all of the discussion about steady detonation wave engines is pure speculation. In this paper, the focus will be on steady waves although it should be pointed out that propagating detonations have been proposed as components in intrinsically unsteady wave engines (Eidelman et al. 1991, Voytsekhovskiy et al. 1964).

In the next section, an elementary review of propagating detonation phenomena will be given. The simple hydrodynamic discontinuity approach to oblique detonation and shock waves is discussed. The idealized structure of the reaction zone for steady oblique waves is then described. This structure is an analog of the standard Zel'dovichvon Neumann-Döring model. Results of numerical solutions to the reaction zone structure using detailed chemical reaction mechanisms are given for some representative cases that are of interest to propulsion. The experimental evidence for steady oblique detonations is presented.

A paradigm of the oblique detonation wave problem is a wave created by the flow deflection around a wedge. The uniqueness and stability of this solution are examined and a possible "test" for detonation-like behavior are proposed. Detonation stability is examined both from the viewpoint of microinstabilities and the potential for catastrophic or global instability of the entire flow. Initiation or failure of the detonation process is related to the presence of transients in the flow that are produced by unsteadiness or flow defiections.

\section{Propagating Detonation Concepts}

Propagating detonations in gases (Strehlow 1984, Fickett and Davis 1979, Zeldovich and Kompaneets 1960) are characterized by a self-sustaining configuration of shock waves and reaction zones, indicated schematically in Fig. 3 for an idealized steady configuration. The propagation velocity $U$ is relatively constant for waves that are self-sustaining and is approximately equal to the ChapmanJouguet $(\mathrm{CJ})$ value $U_{C J}$. The CJ velocity is obtained through purely thermodynamic considerations as the minimum velocity consistent with a steady wave separating reactants and equilibrium products. 
If the combustion process results in the release of energy $q$ per unit mass into the flow, then $U_{C J} \sim \sqrt{2\left(\gamma^{2}-1\right) q}$. The flow following the detonation can then be treated using the standard techniques of nonreactive compressible flow. This flow often consists of a transient expansion wave which eventually brings the fluid back to rest.

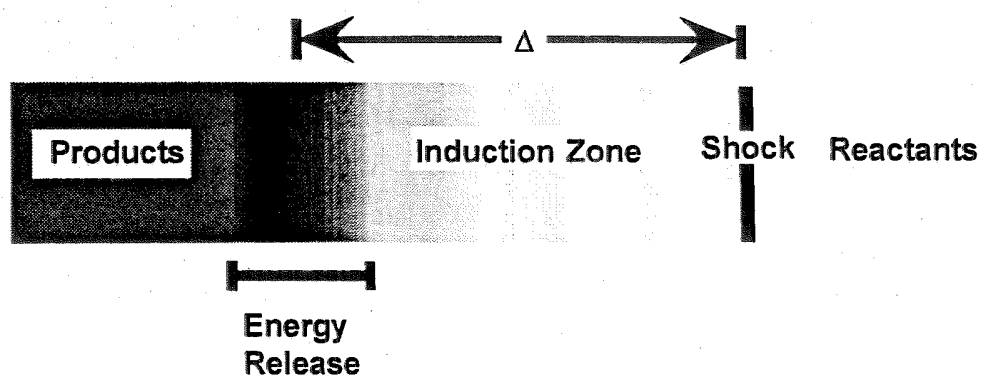

Figure 3: Idealized one-dimensional, steady-state detonation wave configuration (ZND model) consisting of shock wave followed by a reaction zone. The characteristic reaction zone length $\Delta$ is based on the location of the maximum in the chemical energy release rate.

The dynamic behavior of detonation waves is determined by the response of what is usually a relatively small portion of the flow, the shock wave-reaction zone complex located at the front of the wave. The intimate coupling between the shock wave and the chemical reactions occurring in the region immediately behind the shock plays a key role in this response. The shock wave produces the adiabatic compression which increases the gas temperature sufficiently to initiate the chemical reactions that result in the release of energy that drives the shock forward. This cycle of events is unstable since typical chemical reaction rates for hydrogen or hydrocarbon-air mixtures have an extreme sensitivity to the temperature. Small variations in the shock strength produce large variations in reaction rates in the flow directly behind the shock. After some time delay associated with the cumulative effects of chemical reaction and acoustic propagation of disturbances, the changes in reaction rates then result in variations in the shock strength since the flow is subsonic (relative to the shock) through most of the reaction zone. This creates an unstable feedback loop that results in the spontaneous and nonlinear 
instability of propagating detonations in gases.

This instability results in the breakdown of the idealized onedimensional structure shown in Fig. 3, the production of transverse shock waves, an oscillatory motion of the main shock front and a turbulent flow field behind the detonation as shown in Fig. 4. A consequence of this almost universal instability of self-sustaining detonation waves in gases is the formation of quasi-periodic instability patterns associated with the motion of the intersections or triplepoints between the transverse waves and the main front. The cellular appearance of these patterns motivated the term "cellular structure" for the instability and the characteristic transverse wavelength of the instability is referred to as the "cell width". The cell width $\lambda$ is often used (Lee 1984) as length scale that defines an effective thickness of the detonation front. Another commonly used length scale is the calculated thickness $\Delta$ of an idealized one-dimensional, steady reaction zone structure.

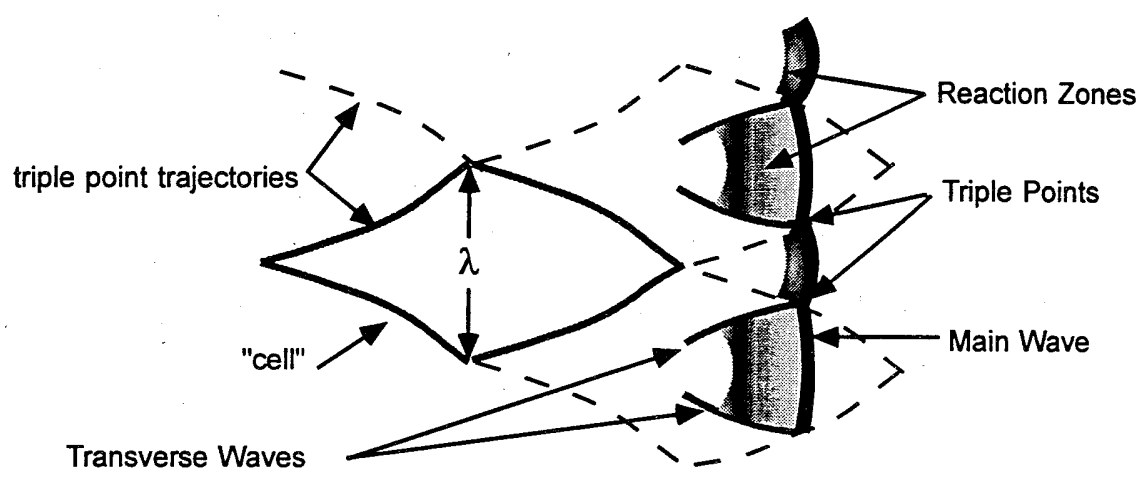

Figure 4: Instability pattern associated with propagating detonations, illustrating cellular pattern formation and characteristic cell width $\lambda$.

The existence of such a macroscopic intrinsic length scale $\lambda$ or $\Delta$ distinguishes detonations from simple shock waves and other nonreactive fronts or interfaces. The processes of detonation initiation, stability of propagation, and behavior during transients such as diffraction can be correlated (Lee 1984) on the basis of relationships of these length scales to the characteristic physical dimensions of the confining boundaries. The correlations indicate that for each mixture 
composition and initial conditions there exist a set of critical length scales that define the limits within which propagating detonations can be produced. Representative configurations that have critical length scales are sketched in Fig. 5.

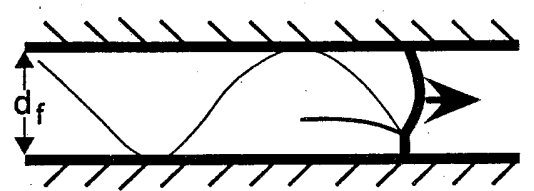

(a)

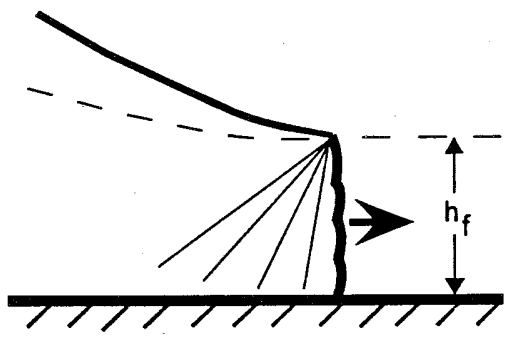

(c)

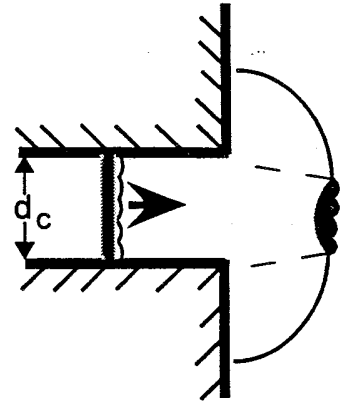

(b)

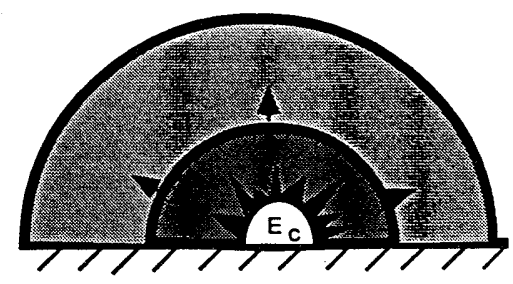

(d)

Figure 5: Configurations associated with critical length scales for detonation behavior. a) failure diameter $d_{f}$ associated with a minimum tube size for confined steady propagation. b) critical tube or orifice diameter $d_{c}$ associated with diffraction of a detonation. c) critical layer thickness $h_{f}$ associated with steady unconfined propagation. d) Critical initiation energy $E_{c}$ associated with the direct initiation of detonation by strong blast waves. 
An alternative to correlating the behavior based on the experimentally measured cell width is to use the computed reaction zone length as based on the idealized ZND model of detonations. Extensive computations and comparisons with experiment (Westbrook and Urtiew 1982, Shepherd 1986) indicate that such a correlation is indeed feasible. Fig. 6 demonstrates the scaling relationships that have been determined in this fashion. Note that the scaling length for initiation is defined as $R_{c}=\left(E_{c} / P_{o}\right)^{1 / 3}$.

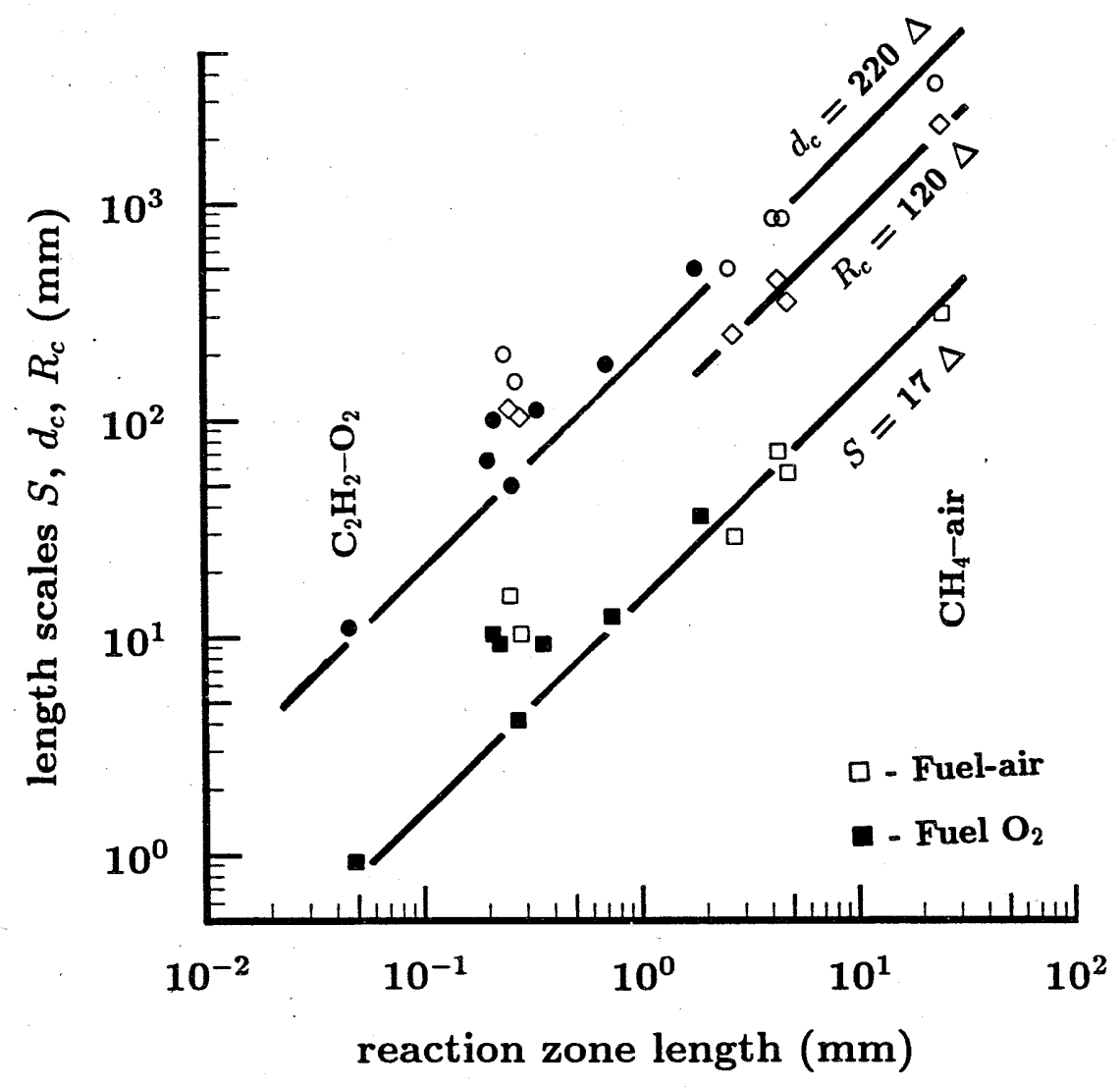

Figure 6: Scaling relationships between the computed ZND reaction zone length and the critical length scales for detonation propagation. The length scale $S$ is the estimated cell width $\lambda$.

Such correlations are based on extensive experimentation and naive dimensional analysis. This simple idea is quite successful in correlating many overall trends but often fails to capture the nu- 
ances of behavior resulting from the interplay between fluid dynamics, chemical reactions and thermodynamics. On the other hand, direct numerical simulations are a powerful tool (Bourlioux and Madja 1992) but at present are only able to examine the simplest models of chemical reaction if the spatial resolution needed for resolving the instability is used.

What is the significance of these critical length scales to propulsion systems and oblique detonation waves in general? Extrapolating from propagating to stationary waves, we conclude that it will only be possible to observe detonation-like behavior under certain specific conditions. It is important to distinguish between conditions sufficient for existence of waves and those conditions required to initiate waves. First, the confining boundaries or the thickness of the combustible layer must exceed a certain minimum size in order that the detonation wave can exist. Second, a minimum amount of energy must be provided over some time period in order to initiate the detonation.

A certain difficulty immediately arises in attempting to extend notions concerning propagating detonations to stationary waves. How do we distinguish stationary detonations from mere shock-induced combustion? Is there a continuous spectrum of behavior or can distinct regimes be identified? I believe that the extreme sensitivity of most chemical reaction rates to temperature will in fact, result in a sharp distinction between the two phenomena: detonations vs. shock-induced combustion.

For propagating waves, the distinction between detonation and shock-induced combustion is clear when the reaction zone terminates in a sharp reaction front or explosion locus. This is the case for most gaseous reactions described by an Arrhenius rate law with at least a modest activation energy. In a detonation wave, the shock and reaction fronts must propagate at essentially the same velocity in order to be coupled. For self-sustaining waves, it is further observed that the wave speed is close to the CJ velocity and the front is unstable to transverse disturbances. In shock-induced combustion, the shock front and reaction front do not travel at the same speed and often become completely uncoupled, that is, the reaction front progressively lags behind the shock front as both waves decay. Shock-induced combustion is a transient process that either terminates in an explosive instability leading to transition to detonation or the decay of the wave system into a low-speed flame with a weak precursor shock 
wave.

Defining steady oblique detonations is apparently a more subtle problem than for propagating waves. For a steady flow, various wave configurations are possible depending on the starting and boundary conditions. In particular, the equivalent wave velocity does not have a unique minimum for steady flows. Many researchers have labeled any supersonic flow process that results in a shock wave followed by a reaction zone an "oblique detonation wave". Is there a simple way to distinguish oblique detonations? I suggest that there are two key tests. First, an oblique detonation must be a wave-like structure that can be enclosed by a control volume and satisfies the usual jump conditions relating upstream and downstream states. Second, the wave should be capable of self-sustained propagation, i.e., if the incoming flow was somehow stopped, the wave would propagate upstream. A clear symptom of the existence of a detonation is the presence of transverse waves. However, in the case of an oblique detonation, these waves may be suppressed if the detonation is sufficiently overdriven.

\section{Oblique Detonation Concepts}

Oblique detonations can be analyzed at several levels. The simplest type of analysis is to treat the detonation wave as a discontinuity analogous to the standard treatment of oblique shock waves (Thompson 1972). This is an essentially thermodynamic analysis that neglects the chemical reaction process and the structure of the detonation wave. A discontinuity analysis determines the locus of possible downstream equilibrium states for a given upstream state. The next more detailed level of analysis is to consider the reaction zone structure of a steady, oblique wave. This structure can be obtained as a transformation of the standard, one-dimensional ZND model of steadily propagating detonations.

The most complex situation for which some analytical considerations can be given is for a weakly-curved wave and the influence of curvature on the existence of detonation-like wave structures. Open questions about the stability and initiation of detonations in combustor geometries can probably only be addressed through experimentation or numerical simulations. The intrinsically multidimensional and transient nature of these processes indicates that direct numerical solutions of the equations of motion are necessary. It is possible 
to obtain some information by solving the simpler problem of computing the normal mode solutions to the linearized stability problem. Although quite well understood (Lee and Stewart 1990) for the case of one-dimensional disturbances, the stability of detonation waves to multi-dimensional disturbances is not well characterized. The linearized stability of oblique detonation waves is not at all understood.

\subsection{Discontinuity Analysis}

The discontinuity model is a purely hydrodynamic construct that considers the upstream state as a specified mixture of reactants and the downstream state as reaction products in a state of chemical equilibrium. An analysis of the conservation laws using a control volume across the wave front (Thompson 1972) yields a locus of solutions known as the Hugoniot relation or the detonation adiabat. Chemical reaction rate considerations, wave structure or instabilities are neglected in this type of analysis. The flow is considered to be steady and all front structure is considered to be contained within the control volume.

Detonation adiabats are essentially one-dimensional concepts that can be calculated without considering the oblique nature of the wave. Transforming to oblique wave coordinates then provides certain restrictions on the possible flow deflection angles as a function of the upstream flow state. This is usually represented in pressure-flow deflection coordinates as a detonation polar. There have been many studies of the detonation polars and the implications for oblique detonation waves, a review of this prior work is provided by Pratt et al. (1991). An outline of the analysis and its implications are given below.

Application of the integral balances for mass, momentum and energy within a thin control volume enclosing a section of the wave of Fig. 2 leads to a set of relationships between the upstream and downstream states across the oblique detonation wave. These relationships are conventionally referred to as jump conditions since a detonation or shock appears to be a sharp jump in properties according to the hydrodynamic discontinuity description. In terms of the mass density $\rho$, normal velocity $w$, tangential velocity $v$, pressure $P$ and specific enthalpy $H$, the results are:

$$
\rho_{1} w_{1}=\rho_{2} w_{2}
$$




$$
\begin{aligned}
P_{1}+\rho_{1} w_{1}^{2} & =P_{2}+\rho_{2} w_{2}^{2} \\
v_{1} & =v_{2} \\
H_{1}+\frac{w_{1}^{2}}{2} & =H_{2}+\frac{w_{2}^{2}}{2}
\end{aligned}
$$

Given a set of upstream conditions (state 1 ) these equations can be solved once an equation of state is selected. The approximation of a mixture of ideal gases with realistic specific heats and heats of formation appears adequate for all air-breathing propulsion applications. The composition, given by amount of each species, is specified for the upstream state and must be computed for the downstream state. For a nonreactive shock wave, the composition is frozen, i.e., will not change across the wave. For the hydrodynamic model of detonation, the composition must be computed by solving the conditions for chemical equilibrium in the products.

Figure 7 shows a typical equilibrium detonation adiabat together with a shock adiabat. These curves show the relationship between pressure and specific volume obtained by eliminating velocity from the jump conditions, Eqns. 1-4, to obtain the Hugoniot relation

$$
H_{2}-H_{1}=\left(P_{2}-P_{1}\right)\left(V_{2}+V_{1}\right) / 2
$$

where $V=1 / \rho$ is the specific volume.

The frozen shock adiabat curve corresponds to the locus of possible states behind a non-reacting shock wave for upstream conditions. Each point corresponds to a given upstream normal velocity. The equilibrium shock adiabat curve is similar except that the points now correspond to the various equilibrium states attainable behind the detonation wave given the upstream thermodynamic condition, composition, and velocity. These results were obtained by numerical solution (Reynolds 1986) of the jump conditions with realistic thermodynamic properties and a full set of reaction products in the case of the equilibrium model. The CJ detonation velocity is about 1908 $\mathrm{m} / \mathrm{s}$ for this mixture. Note that the detonation adiabat is substantially different than the usual rectangular hyperbola that results if the specific heat and heat of reaction are taken to be constant. The equilibrium and frozen solution curves cross when the normal velocity $w_{1}$ reaches about $3350 \mathrm{~m} / \mathrm{s}$. This is a consequence of the dissociation of the reaction products that occurs at high temperatures; the products actually have to cool down to reach equilibrium. This may 
play a role in limiting the operational regime for a detonation-based propulsion system.

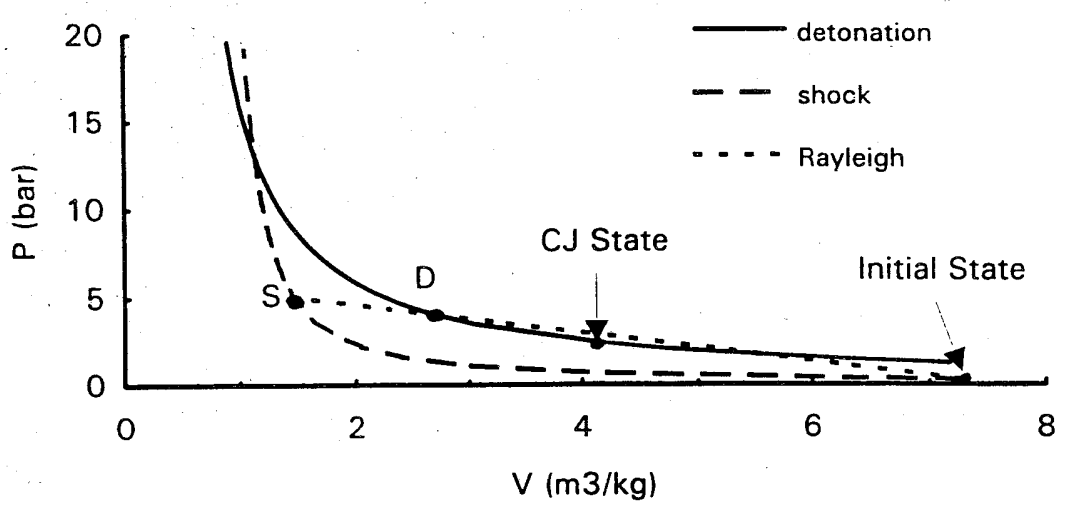

Figure 7: Shock and detonation adiabats for a stoichiometric hydrogen-air mixture with upstream conditions of $550 \mathrm{~K}$ and $.3 \mathrm{bar}$, appropriate to the exit of the inlet diffuser for a detonation-based propulsion system.

\subsection{ZND Model}

The relevance of the shock adiabat is thus: An idealized model of the detonation structure can be formulated by supposing that a nonreactive shock precedes any chemical reactions. The basis for this idea is the notion that a shock occurs over only a few collision distances (mean free paths) of the reactant molecules while significant chemical reaction requires thousands or millions of collisions. This is the model first proposed by Zel'dovich, von Neumann and Döring (ZND model) to explain the role of shock waves and chemical reaction in detonation propagation.

During a steady reaction process, conservation of mass and momentum require the $P-v$ locus to lie on a straight line:

$$
P_{2}-P_{1}=-\left(\rho_{1} w_{1}\right)^{2}\left(V_{2}-V_{1}\right)
$$

These lines are drawn on Fig. 7 and are known as Rayleigh lines. The thermodynamic path from the initial state to the final equilibrium 
state can be conveniently traced out in Fig. 7. The fluid initial state is the low pressure and density condition at the lower right. The nonreactive shock preceding the reaction zone corresponds to moving along the Rayleigh line up to the frozen shock adiabat. As the fluid reacts, the state moves down along the Rayleigh line from the frozen shock adiabat to the point of intersection with the equilibrium shock adiabat. Note that the Rayleigh line will always have two intersections with the equilibrium shock adiabat except in the limiting case when the Rayleigh line is actually tangent to the equilibrium shock adiabat.

The point of tangency corresponds to the minimum upstream flow velocity that is required to reach the equilibrium shock adiabat. This minimum velocity is referred to as the Chapman-Jouguet velocity $\left(w_{C J}\right)$, and is only a function of the mixture composition and the conditions at the upstream state. Rayleigh lines corresponding to overdriven detonation waves have an upstream velocity larger than the CJ value, $w>w_{C J}$. As long as the amount of overdrive is not excessive, the equilibrium shock adiabat is to the right of the frozen one and the temperature is higher in the equilibrium products than in the shocked reactants. If the detonation velocity is high enough, the postshock state will lie above the point where the adiabats cross and product temperature will be lower than the shocked fluid temperature.

If the upstream velocity is less than the CJ value, then apparently a steady detonation solution leading to complete combustion does not exist. There are two possibilities in this case. An unsteady flow leading to complete combustion consists of a shock wave followed by a slower reaction wave, termed shock-induced combustion. This configuration is intrinsically unsteady since the reaction wave progressively lags behind the shock in this situation (Zeldovich and Kompaneets 1960). The usual consequence of this unsteadiness is the decay of the shock wave and the eventual conversion of the reaction wave into a low-speed flame. Such a process may also be unstable since an explosion within the region behind the shock and the reaction wave will produce pressure waves that influence the leading shock. This can lead to a pulsating type of instability or transition to detonation in the case of a propagating detonation (Bach et al. 1969).

A steady but incomplete combustion process can also occur behind a steady shock wave that is curved (Bdzil and Stewart 1989). 
The flow divergence behind the wave competes with the chemical reaction and results in a sonic point before the reaction is complete. This type of solution plays an important role in determining the combustion process produced behind the bow shock of a hypersonic projectile in a reacting gas. These solutions exist only if the wave curvature is not too large, which sets conditions on the existence of curved detonation waves.

The Rayleigh line shown in Fig. 7 corresponds to an overdriven wave with a normal velocity $w_{1}=2086 \mathrm{~m} / \mathrm{s}$, about $10 \%$ higher than the CJ velocity. The figure shows two possible intersections between the Rayleigh line and the detonation adiabat. In principle, both intersection points are possible solutions: a "strong" one in which the flow behind the wave is subsonic, and a "weak" one in which the flow behind the wave is supersonic. In order to determine which of these solutions are actually realized, further considerations of the details of the chemical reaction mechanism are required. The supersonic solution is usually ruled out by these considerations since it is only possible in exceptional cases to smoothly pass through the sonic point that separates the two types of solutions (Fickett and Davis 1979). This is discussed in the subsequent section on reaction zone structure.

\subsection{Application to Oblique Waves}

An oblique detonation can be treated as a normal detonation by considering an orthogonal set of axes in the which the wave lies along one of the axes. In this case, only the normal component of velocity plays a role in the solution. According to the previous discussion of the jump conditions, the tangential velocity component remains unchanged. Note that for oblique detonation waves, the minimum upstream velocity requirement is now stated in terms of the normal component of the velocity, $w_{1} \geq w_{C J}$. From the geometry of Fig. 2, the equations for the upstream and downstream normal velocity components are given by:

$$
\begin{aligned}
& w_{1}=u_{1} \sin \beta \\
& w_{2}=u_{2} \sin (\beta-\theta)
\end{aligned}
$$

The net velocities upstream and downstream of the wave are $u_{1}$ and $u_{2} ; \beta$ is the detonation wave angle, and $\theta$ is the flow deflection angle. The tangential component of velocity, $v$, 


$$
v=u_{1} \cos \beta=u_{2} \cos (\beta-\theta)
$$

is constant across the wave, which implies that:

$$
w_{2}=u_{1} \cos \beta \tan (\beta-\theta)
$$

Combining this last relation with the geometric transformations and the solutions to the normal detonation wave problem $w_{2}=f\left(w_{1}\right)$ discussed earlier, the wave angle $\beta$ and the flow deflection angle $\theta$ can be determined for a given upstream velocity $u_{1}$ and normal velocity $w_{1}$.

$$
\begin{aligned}
& \beta=\sin ^{-1}\left(w_{1} / u_{1}\right) \\
& \theta=\beta-\tan ^{-1}\left(\frac{w_{2}}{\sqrt{u_{1}^{2}-w_{1}^{2}}}\right)
\end{aligned}
$$

Realistic polar curves can be readily computed once the normal shock wave or detonation adiabats have been determined (Sabet 1990). The procedure is to first fit the computed relation between upstream and downstream normal velocity components to a polynomial curve. This relationship can then be used to evaluate $\beta$ and $\theta$ from Eqns. 11 and 12. The other thermodynamic properties downstream of the wave can be determined from the jump conditions, Eqns. 1-4. Maximum flow deflection angle can be computed by first finding an analytical expression for the derivative of the flow deflection angle with respect to the upstream normal velocity. A numerical root-solver can then be used to solve for the value of the upstream normal velocity which makes this derivative zero.

This procedure enables the use of available computational tools for normal waves (Reynolds 1986, Gordon and McBride 1972) to be used to generate solutions for oblique waves without having to make any assumptions regarding the specific heats, energy release or equilibrium compositions. Once a shock adiabat is determined in the form $w_{2}=f\left(w_{1}\right)$, solutions for any upstream velocity can be obtained readily by these simple transformations. Both the frozen and equilibrium states can be treated in this fashion in order to get the oblique analog of the ZND model. In order to complete this picture, the momentum flux conservation equation can be transformed 
to obtain the analog of the Rayleigh line which we term the Rayleigh curve:

$$
P_{2}=P_{1}+\rho_{1} u_{1}^{2} \frac{\tan \theta}{\cot \beta+\tan \theta}
$$

A set of shock and detonation polar curves and a connecting Rayleigh curve are shown in Fig. 8 for a freestream velocity of 2900 $\mathrm{m} / \mathrm{s}$ and the upstream thermodynamic conditions used for the adiabats shown in Fig. 7. The Rayleigh curve shown corresponds to that of the slightly overdriven solution shown in Fig. 7 and results in a wave angle of about $46^{\circ}$. The locus of possible values $\beta$ and $\theta$ for this example is shown in Fig. 9.

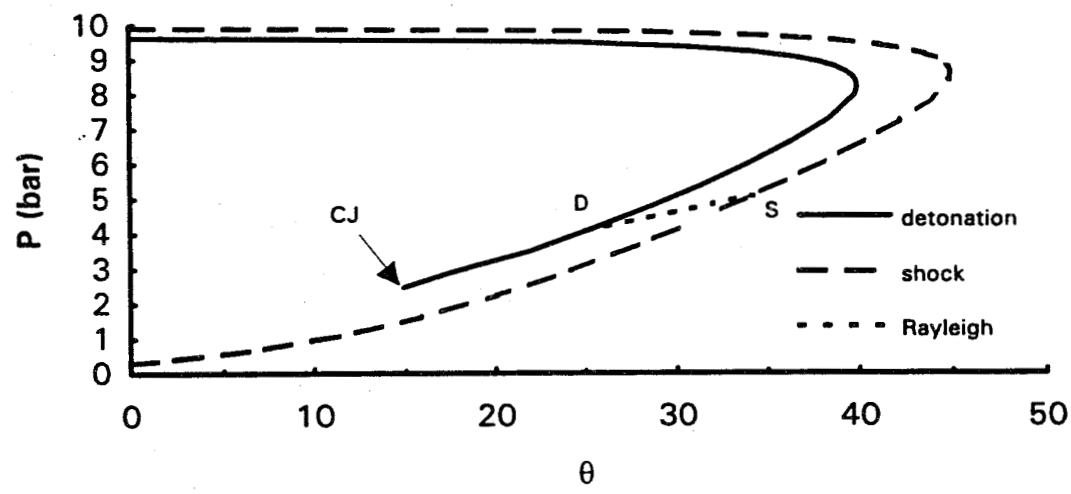

Figure 8: Shock and detonation polar curves for a stoichiometric hydrogen-air mixture with upstream conditions of $550 \mathrm{~K}, .3 \mathrm{bar}$ and $2900 \mathrm{~m} / \mathrm{s}$. The Rayleigh curve for a idealized detonation structure is shown connecting the postshock and equilibrium states for a wave angle $\beta$ of $46^{\circ}$.

Key points to note are the minimum $\theta_{C J}$ and maximum $\theta_{\max }$ flow deflection angles associated with transformation between uniform upstream and downstream states. These are only local limits on flow deflections. If the flow downstream must ultimately deflect outside these ranges, then there will be a nonuniform flow following the detonation and/or the detonation wave will be curved. The nature of the solutions in those cases will be discussed in a subsequent section. 
A pair of representative streamlines and the flow deflection within. an exothermic reaction zone is sketched in Fig. 10. This would be characteristic of the solution moving along the Rayleigh curve indicated in Fig. 8 for a solution with a slight amount of overdrive. The flow deflection history can be readily deduced from the structure equations given in the subsequent section. After passing through the shock, $\theta$ jumps to the value $\theta_{S}$. As the chemical reaction proceeds, the flow deflects away from the wave for exothermic reactions and toward the wave for endothermic reactions. Ultimately, $\theta$ approaches the value $\theta_{D}$ as the reactions come to equilibrium.

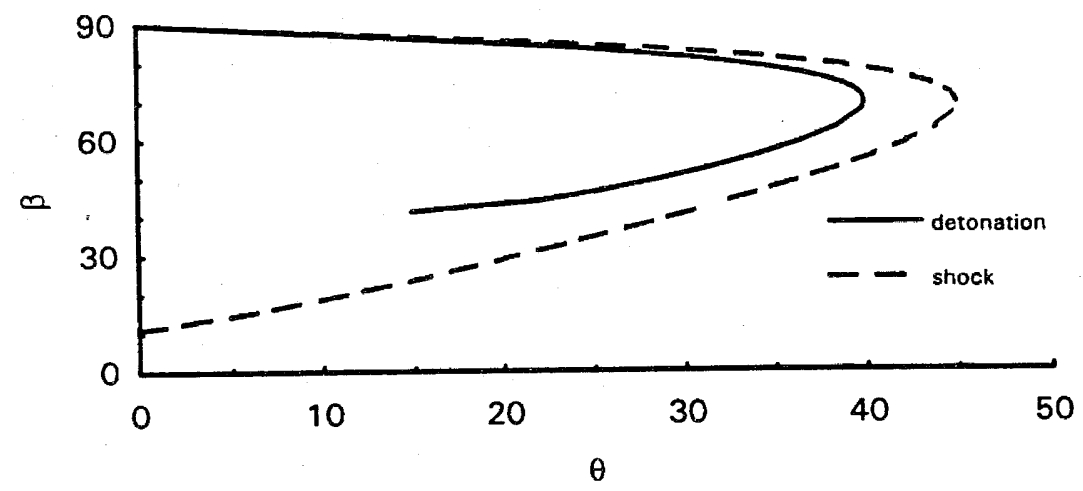

Figure 9: Possible range of wave angle $\beta$ and flow deflection angle $\theta$ for an oblique detonation in a stoichiometric hydrogen-air mixture with upstream conditions of $550 \mathrm{~K}, 0.3 \mathrm{bar}$ and $2900 \mathrm{~m} / \mathrm{s}$.

As the amount of overdrive becomes larger, $w_{1} \gg w_{C J}$, endothermic processes will become more significant and the flow deflection will be nonmonotonic within the reaction zone. If the overdrive is sufficiently large, the VN point will lie beyond the point where the two adiabats cross and the flow deflection throughout the reaction zone will be in the opposite sense to that shown in Fig. 10. In any case, the state of the flow will move along the Rayleigh curve, approaching the equilibrium adiabat as the reactions come to equilibrium in the flow behind the shock wave. 


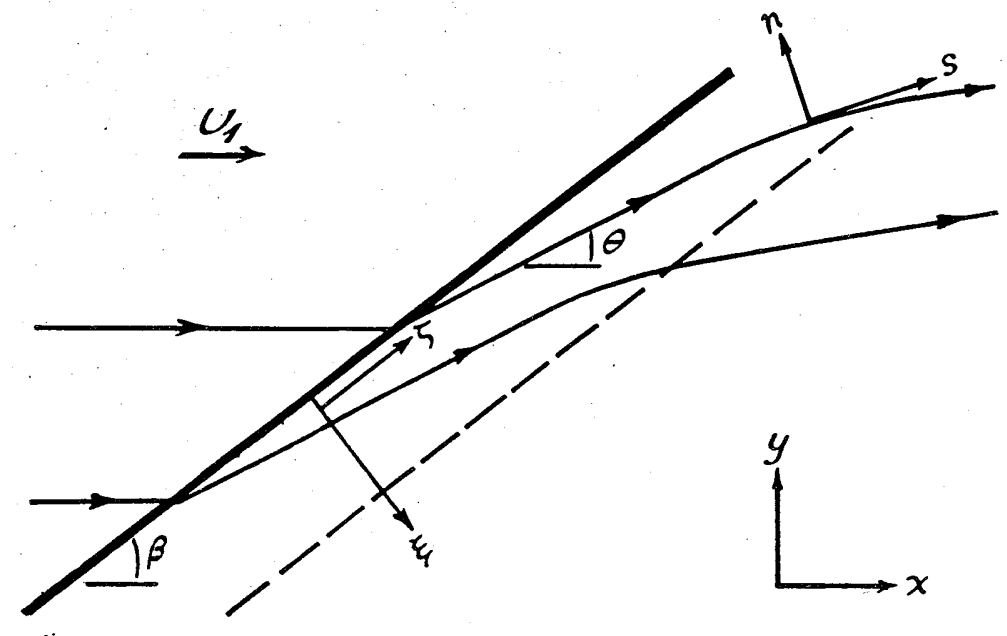

Figure 10: Flow deflection along a typical streamline with exothermic reactions within the reaction zone of an idealized oblique detonation. The natural $(n, s)$ and wave-fixed $(\zeta, \xi)$ coordinates are also shown.

\subsection{Reaction Zone Structure Equations}

The flow within the reaction zone of an oblique detonation wave can be analyzed in more detail by considering the equations of motion for an inviscid, reacting, compressible flow. For two-dimensional flow, it is convenient to utilize a curvilinear system of coordinates as shown in Fig. 10. In such a system, one axis $(s)$ is along the streamline, and the other axis $(n)$ is orthogonal to it. Instead of considering two velocity components, we use the magnitude of the velocity $u$ and the deflection angle $\theta$ relative to a reference axis. The steady conservation equations in this frame of reference are (Liepmann and Roshko 1957) for mass:

$$
\frac{\partial(\rho u)}{\partial s}=-\rho u \frac{\partial \theta}{\partial n}
$$

momentum components: 


$$
\begin{aligned}
\rho u \frac{\partial u}{\partial s} & =-\frac{\partial P}{\partial s} \\
\rho u^{2} \frac{\partial \theta}{\partial s} & =-\frac{\partial P}{\partial n}
\end{aligned}
$$

energy:

$$
H+\frac{u^{2}}{2}=H_{0}
$$

and species:

$$
\rho u \frac{\partial Y_{k}}{\partial s}=W_{k} \dot{\omega}_{k} \quad k=1, \ldots, K
$$

where $Y_{k}$ is the mass fraction of species $k, W_{k}$ is the molar mass of species $k$, and $\dot{\omega}_{k}$ is the net molar production rate of species $k$. A chemical reaction mechanism and a set of associated rate constants is required to compute the net molar production rate $\dot{\omega}_{k}$ of species $k$. The total enthalpy $H_{o}$ has a constant value for a given streamline but may vary if the upstream state is nonuniform.

For a reacting flow, the fundamental property relation of thermodynamics is

$$
d H=T d S+V d P+\sum_{k} \mu_{k} d N_{k}
$$

where $S$ is the specific entropy and $N_{k}$ is the number of mols of species $k$ per unit mass of material and $\mu_{k}$ is the chemical potential or partial Gibbs energy per mol of species $k$. This relationship and the momentum conservation equations can be combined to form the reacting flow extension to the Crocco-Vazsonyi equation (Thompson 1972) for steady, inviscid flows:

$$
T \nabla S+\sum_{k} \mu_{k} \nabla N_{k}=(\nabla \times u) \times u+\nabla H_{o}
$$

We conclude that the entropy changes will only be due to chemical reaction as long as the upstream fluid state is uniform $H_{o}=$ constant and the vorticity $\nabla \times u$ vanishes.

The conditions of Crocco's theorem will be satisfied by a straight, oblique detonation wave extending into a uniform flow. The direct relationship between entropy changes and species changes under these 
circumstances enables the substitution of the energy equation with the adiabatic change equation (Fickett and Davis 1979):

$$
d P=a^{2} d \rho+\rho a^{2} \sum_{k} \sigma_{k} d Y_{k}
$$

where $a$ is the local (frozen) speed of sound,

$$
\left.a^{2} \equiv \frac{\partial P}{\partial \rho}\right)_{S, Y_{k}}
$$

The thermicity components $\sigma_{k}$ are the nondimensional energy release associated with production of species $k$. For an ideal gas mixture, these components are:

$$
\sigma_{k}=\frac{W}{W_{k}}-\frac{H_{k}}{C_{p} T}
$$

where $W$ is the mixture average molar mass. The thermicity components are the coupling coefficients that determine the interaction between chemical reaction and gasdynamics. The first term represents the contribution of the changes in the number of mols and the second term represents the changes in the enthalpy of the mixture. Net exothermic and mol producing reactions result in positive values of $\sigma_{k}$, net endothermic and mol reducing reactions have negative values of $\sigma_{k}$. Note that the adiabatic change equation is the reacting flow extension of the isentropic relationship $d P=a^{2} d \rho$ that is frequently used in nonreacting compressible flow analyses.

Vorticity can be expressed in natural coordinates as

$$
\nabla \times u=u \frac{\partial \theta}{\partial s}-\frac{\partial u}{\partial n}
$$

so that the irrotational flow condition $\nabla \times u=0$ will be

$$
u \frac{\partial \theta}{\partial s}=\frac{\partial u}{\partial n}
$$

Further, if wave front is straight, then there is translational invariance along the wave and $\partial / \partial \zeta=0$, which yields:

$$
\frac{\partial}{\partial n}=\frac{-1}{\tan (\beta-\theta)} \frac{\partial}{\partial s}
$$


Combining the conservation equations with the irrotational and translational invariance conditions results in the reaction zone structure equations in natural coordinates:

momentum:

$$
\rho u \frac{\partial u}{\partial s}=-\frac{\partial P}{\partial s}
$$

continuity:

$$
\frac{\partial(\rho u)}{\partial s}=\frac{\rho u}{\tan (\beta-\theta)} \frac{\partial \theta}{\partial s}
$$

irrotational:

$$
u \frac{\partial \theta}{\partial s}=\frac{-1}{\tan (\beta-\theta)} \frac{\partial u}{\partial s}
$$

adiabatic:

$$
\frac{\partial P}{\partial s}=a^{2} \frac{\partial \rho}{\partial s}+\rho a^{2} \sum_{k} \sigma_{k} \frac{\partial Y_{k}}{\partial s}
$$

species:

$$
\rho u \frac{\partial Y_{k}}{\partial s}=W_{k} \omega_{k} \quad k=1, \ldots, K
$$

In general, a numerical solution of structure equations will have to be obtained if realistic reaction mechanisms and rates are considered. However, for a straight oblique wave, the natural coordinate formulation is unnecessarily complex. A simpler formulation can be obtained by incorporating the invariance of the transverse velocity $v$. Then we only have to consider variations in the flow velocity $w$ normal to the wave. This is facilitated by defining the Mach number $M_{n}$ of the flow normal to the wave front

$$
M_{n}=M \sin (\beta-\theta)
$$

where $M=u / a$ and the sonic parameter $\eta$ is

$$
\eta=1-M_{n}^{2}
$$

It is also conventional to carry out the numerical integration with Lagrangean time $\tau$ as the independent variable rather than the spatial 
coordinate. The conversion between Lagrangean time and distance along the streamlines is

$$
\frac{d s}{d \tau}=u
$$

The contribution of all the thermicity terms can be represented as a sum

$$
\dot{\sigma}=\sum_{k} \sigma_{k} \frac{d Y_{k}}{d \tau}
$$

The Lagrangean-time version of the structure equations are:

$$
\begin{aligned}
\frac{d P}{d \tau} & =-\rho w^{2} \frac{\dot{\sigma}}{\eta} \\
\frac{d \rho}{d \tau} & =-\rho \frac{\dot{\sigma}}{\eta} \\
\frac{d Y_{k}}{d \tau} & =W_{k} \frac{\dot{\omega}_{k}}{\rho} \quad k=1, \ldots, K
\end{aligned}
$$

The initial conditions for these equations are found by solving the jump conditions for the state on the frozen shock adiabat.

The question of flow deflection can be examined by combining these equations to obtain

$$
\frac{\partial \theta}{\partial s}=-\sin 2(\beta-\theta) \frac{\dot{\sigma}}{\eta}
$$

For the situation shown in Fig. 2, the argument of the sine function is positive and less than $\pi$. The rate of change of $\theta$ along the streamline is therefore opposite in sign to the thermicity $\dot{\sigma}$. This supports the previous assertion that the flow will turn away from the wave for an exothermic reaction and toward the wave for an endothermic reaction when the component of the flow normal to the wave is subsonic. This is always the case for the initial portion of the reaction zone behind a shock.

Note that these equations are identical to those (Fickett and Davis 1979) for steadily-propagating detonations with the idealized ZND structure. This establishes the correspondence between the reaction zone structure of idealized oblique waves and normallypropagating waves. The reaction zone structure of a planar, oblique wave is just that of the planar, normal wave rewritten in terms of 
coordinates relative to the wave front. Note that the introduction of upstream disturbances (Jackson, et al. 1990, Lasseigne and Hussaini 1993) or the curvature of the wave front (Bdzil and Stewart 1989) will invalidate this correspondence. In the case of a weakly-curved wave, an additional term can be introduced into the continuity equation to describe the effect of curvature on the flow within the reaction zone.

The known results (Fickett and Davis 1979) about the existence of solutions to the structure equations for the normally propagating wave can now be applied to the oblique wave problem. In particular, it is known that the minimum normal velocity $w_{1}$ for which solutions exist may be determined by the vanishing of the sonic parameter $\eta$ rather than the CJ condition. Note that behind a shock wave, the normal component of the flow is always subsonic so that $\eta$ will start out positive. If the reactions are exothermic then $P$ and $\rho$ will both decrease with increasing $\tau$ and vice versa for endothermic reactions. For exothermic reactions, the normal component Mach number will increase with increasing $\tau$ so that $\eta$ will decrease and can approach zero as the flow comes to equilibrium. In order that the reaction zone structure equation solutions not be singular then $\dot{\sigma}=0$ if $\eta=$ 0 within the reaction zone. In general this will only occur for one particular value of the normal velocity, $w_{\min }$.

If the reaction mechanism consists of an initially exothermic process followed by endothermic reactions, then the minimum normal velocity $w_{\min }$ will be larger than the CJ velocity. Effectively, only the energy released before the sonic point is added to the flow and plays a role in determining the minimum wave speed. For typical fuel-air mixtures, the computed minimum velocity is only slightly larger (3 to $7 \%$ ) than the CJ velocity and the effects of instability and intrinsic unsteadiness mask this from being observed in propagating detonations. The actual observed minimum velocity of propagating detonations is usually 5 to $10 \%$ less than the computed CJ velocity. The is apparently due to the effects of the instability waves and losses to the boundaries. Most studies of detonations neglect the issue of reaction structure and assume that the minimum possible velocity corresponds to the CJ solution to the hydrodynamic model.

Nonsingular solutions with $w_{1}<w_{\min }$ are not possible for steady waves since $\dot{\sigma}$ will not vanish at the same time $\eta$ does. Overdriven solutions $w>w_{\min }$ will terminate with a subsonic normal velocity $1>\eta>0$. In the case of $w=w_{\text {min }}$, the flow can reach a supersonic normal velocity component at the end of the reaction zone by 
passing through the point $\eta=0$. This corresponds to the lower portion of the detonation adiabat, below the CJ point. Reaching this is clearly an exceptional situation and ordinarily only the portion of the detonation adiabat above the CJ point is accessible. This is the reasoning behind terminating the detonation adiabats and polars at the CJ point in the previous discussion of the hydrodynamic model. Introducing wave curvature and upstream disturbances can reduce the minimum allowed velocity, since these influences effectively provide loss mechanisms that reduce the thermicity $\dot{\sigma}$.

\subsection{Reaction Zone Structure Computations}

In order to illustrate the nature of the solutions to these equations, numerical solutions have been computed (Shepherd 1986, Sabet 1990) using a detailed chemical mechanism for hydrogen-air combustion. The LSODE solver package (Hindmarsh 1983) for stiff ordinary differential equations was used together with the CHEMKIN subroutine package (Kee et al. 1990) for the chemical bookkeeping.

The ideal gas equation of state is used:

$$
P=\rho R T \quad R=\tilde{R} / W \quad W=1 / \sum_{k=1}^{K} Y_{k} / W_{k}
$$

where $\tilde{R}$ is the universal gas constant and $W_{k}$ are the molar masses of each species $k$. The mixture specific enthalpy is given by:

$$
H=\sum_{k=1}^{K} Y_{k} H_{k}(T)
$$

where the individual species specific enthalpies $H_{k}$ are computed from the NASA curve fits to the specific heat tabulations and the enthalpy of formation $\Delta H_{f, 298}^{0}$ given in the JANAF compilation (Chase et al. 1985):

$$
H(T)=\Delta H_{f, 298}^{0}+\int_{298}^{T} C_{p}(T) d T
$$

The reaction mechanism used is the standard hydrogen-air mechanism presented in Lutz et al. (1992). The reaction rate equations are of the modified Arrhenius form:

$$
k=A\left[X_{l}\right]^{\nu_{l}}\left[X_{m}\right]^{\nu_{m}} T^{n} \exp \left(-E_{a} / \tilde{R} T\right)
$$


where the pre-exponential term, $A$, the temperature exponent, $n$, and the activation energy, $E_{a}$, are given in Lutz et al. Reverse reaction rates are computed from forward rates by using detailed balancing and equilibrium constants. The molar concentrations of the species are denoted by $\left[X_{i}\right]$, and $\nu_{l}$ and $\nu_{m}$ are the stoichiometric coefficients of the species $x_{l}$ and $x_{m}$ in the elementary reaction formula:

$$
\nu_{l} X_{l}+\nu_{m} X_{m} \longrightarrow \nu_{i} X_{i}+\nu_{j} X_{j}
$$

Contributions of both forward and reverse reactions are included in the computation of the net molar reaction rate for each species.

Examples of the ZND structure for stoichiometric hydrogen-air reaction zones are shown in Fig. 11-13. The initial conditions of 550 $\mathrm{K}, 0.30 \mathrm{~atm}$ and a freestream velocity of $2900 \mathrm{~m} / \mathrm{s}$ corresponds to combustor inlet conditions for a detonation-based propulsion system. The spatial variation of pressure $P$, temperature $T$ and thermicity $\dot{\sigma}$ are shown for three degrees of overdrive $w / w_{C J}=1.09,1.4$, and 1.8.

The reaction zone structure for the near-CJ case consists of a characteristic delay or induction period followed by a rapid release of energy ( water molecules. The temperature and pressure are approximately constant in the induction region. Since the reactions are primarily exothermic, the temperature increases and the pressure decreases with increasing distance through the reaction zone.

The reaction zone structure is significantly different for the highly overdriven cases. The thermicity $\dot{\sigma}$ has several maxima and for large enough overdrive, the pressure will increase and the temperature decrease with increasing distance through the reaction zone. This is due to the increasing importance of dissociation processes with increasing shock normal velocity and consequently, postshock temperature. The first peak in $\dot{\sigma}$ corresponds to the formation of the water molecules. The formation of water in this case does not increase the temperature significantly since the thermal and kinetic components of the energy of the flow are so large that the chemical heat release becomes negligible by comparison. The subsequent decrease in $\dot{\sigma}$ corresponds the formation of intermediates such as $\mathrm{H}, \mathrm{O}$ and $\mathrm{OH}$ which have large positive heats of formation. The second peak in $\dot{\sigma}$ corresponds the leveling off of the concentrations of the $\mathrm{OH}$ radical, a slight decrease in the concentration of the $\mathrm{H}$ and $\mathrm{O}$ atoms, and a slight rise in the concentrations of $\mathrm{H}_{2}$ and $\mathrm{O}_{2}$. 


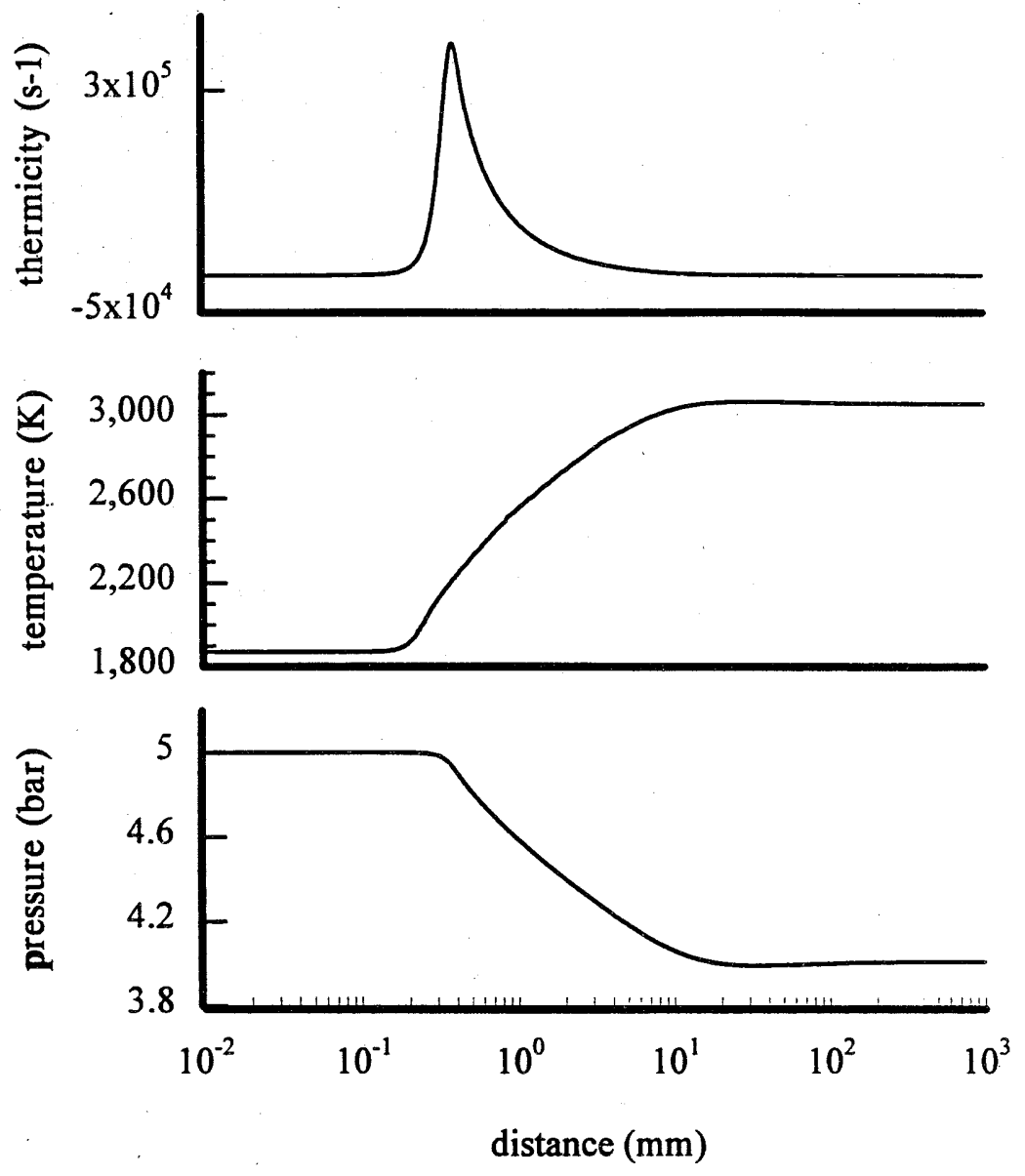

Figure 11: Calculated reaction zone structure for a near-CJ $(w=$ $\left.1.09 w_{C J}\right)$ stoichiometric hydrogen-air detonation with initial pressure of $0.3 \mathrm{bar}$ and a temperature of $550 \mathrm{~K}$. 

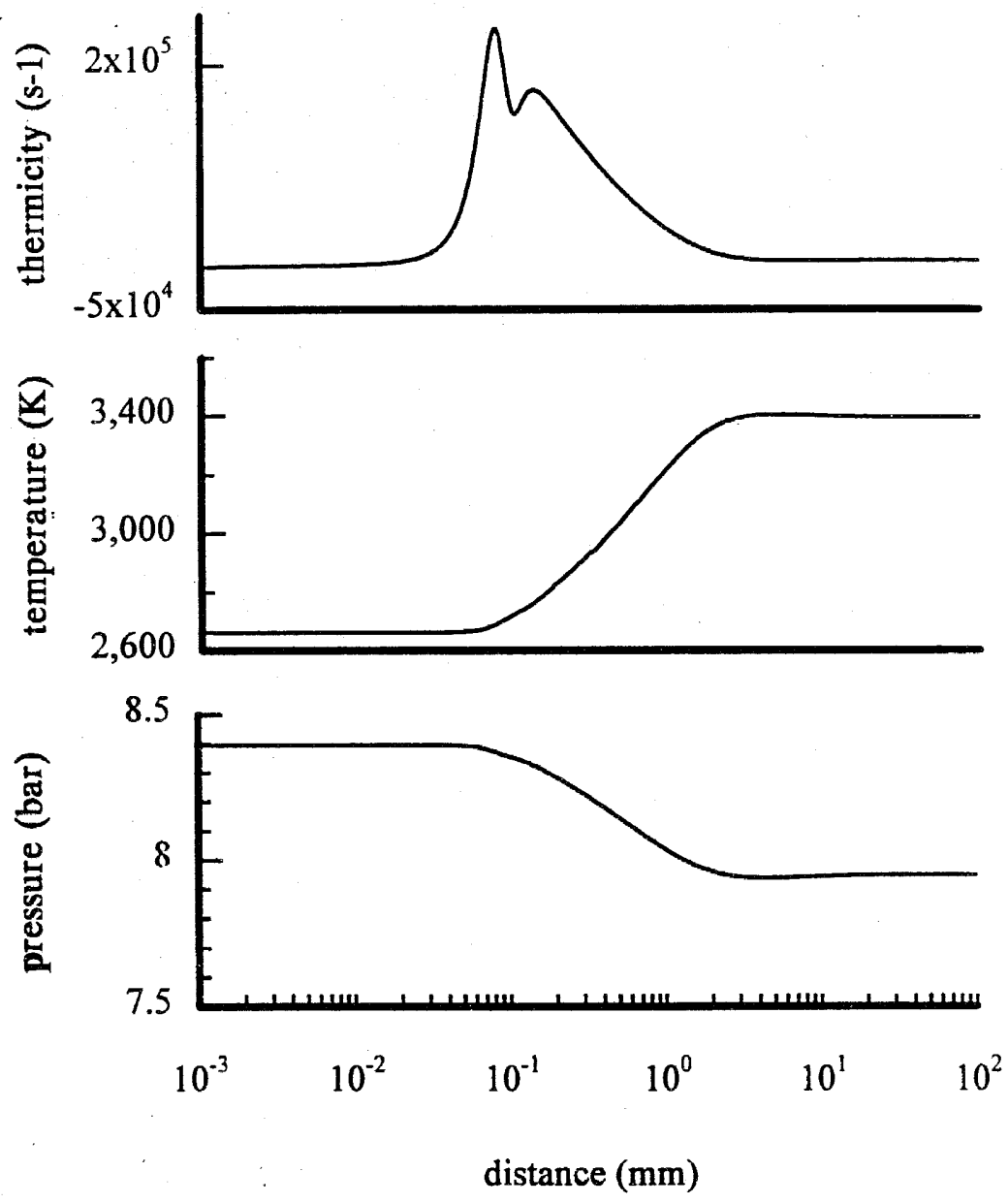

Figure 12: Calculated overdriven $\left(1.4 w_{C J}\right)$ reaction zone structure for a stoichiometric hydrogen-air detonation with initial pressure of 0.3 bar and a temperature of $550 \mathrm{~K}$. 

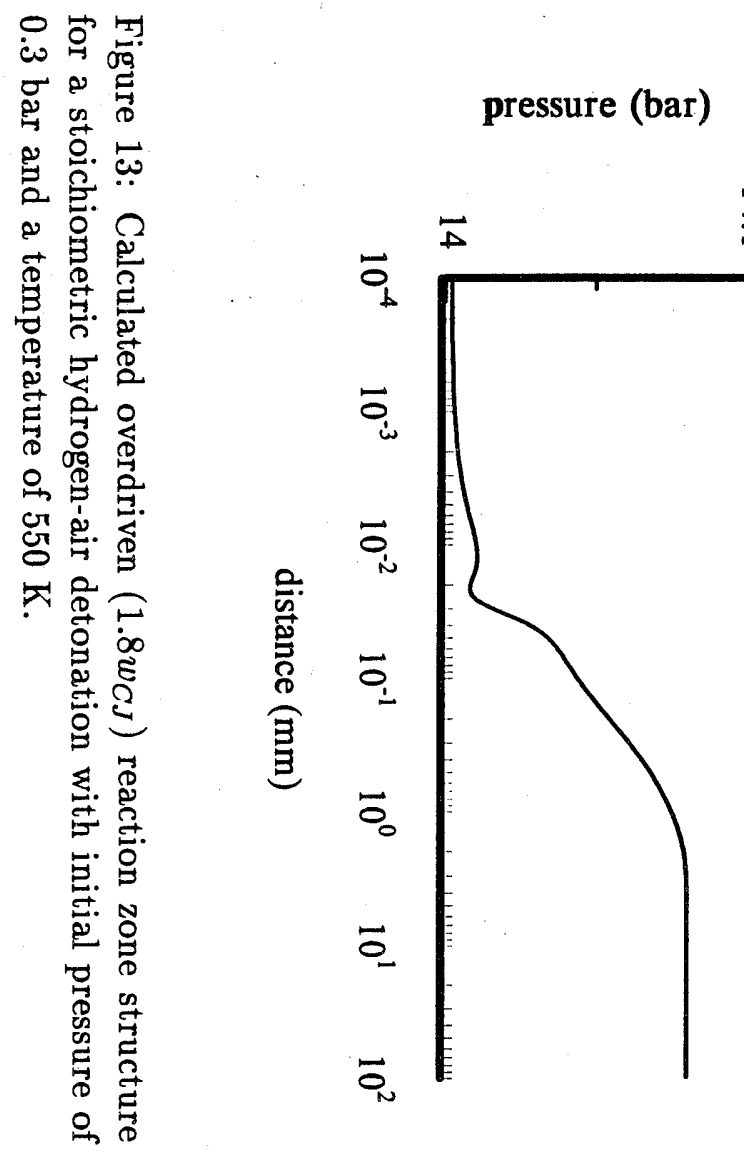

temperature (K)

thermicity (s-1)

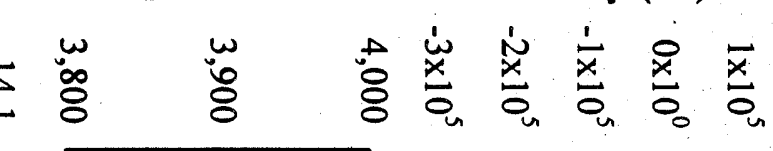

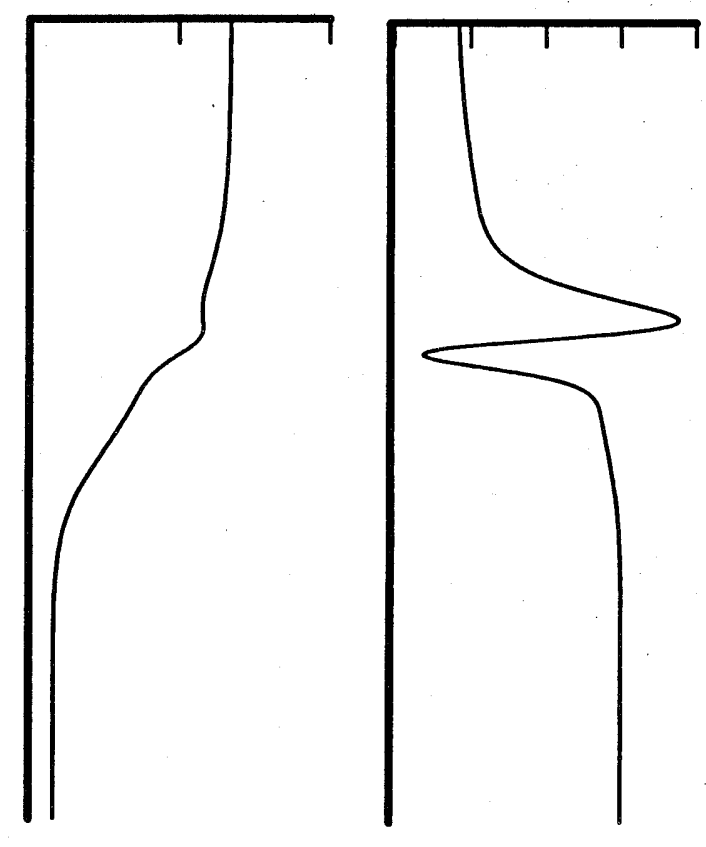




\section{Experiments on "Stationary" Detonation Waves}

Four typical configurations that have been used to examine issues connected to stabilized or "stationary" detonations are shown in Fig. 14. Conceptually, flow over a wedge or a standing wave in a jet or nozzle is the simplest configuration. Early experiments were carried out by Gross and Chinitz (1960) using wedges and Nicholls et al. (1959) using jets. Although the terms "standing detonation wave" are often used in connection with these experiments, very little effect of combustion on the shock wave could be observed (Nicholls 1963) and the terms "shock-induced combustion" are more appropriate.

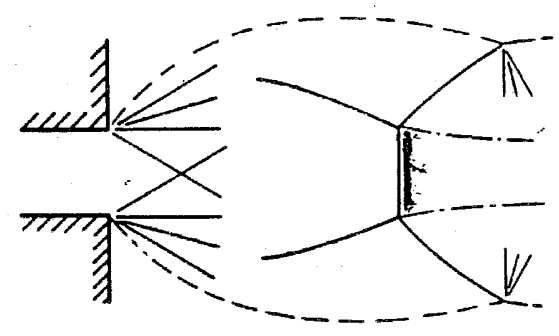

(a)

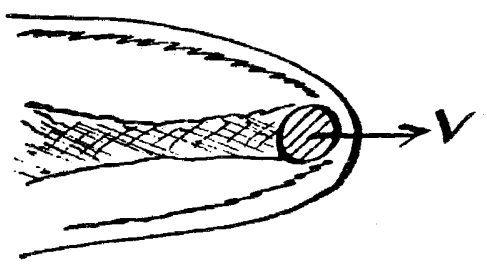

(c)

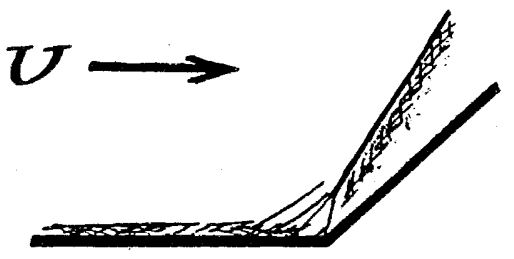

(b)

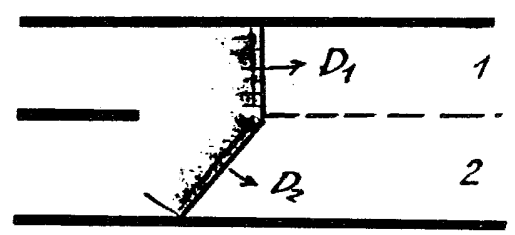

(d)

Figure 14: Four oblique detonation wave configurations. a) underexpanded supersonic jet with a Mach disk. b) wind or shock tunnel flow over a wedge. c) hypersonic projectiles d) two-layer propagating detonations.

The key problem is creating standing detonation waves is the difficulty of obtaining a fuel-air mixture with sufficient stagnation 
enthalpy $H_{o}$ without getting preignition and burning upstream of the shock. A high stagnation enthalpy is needed so that the postshock temperature will be sufficient to initiate rapid chemical reactions and the energy release will couple with the shock front to create the detonation structure. The necessary stagnation enthalpy can be estimated by computing the stagnation states upstream of a normal CJ detonation. For a stoichiometric $\mathrm{H}_{2}$-air mixture, $H_{0} \geq$ $2 \mathrm{MJ} / \mathrm{kg}$ and for stoichiometric $\mathrm{H}_{2}-\mathrm{O}_{2}$ mixture $H_{o} \geq 4.5 \mathrm{MJ} / \mathrm{kg}$. If the stagnation enthalpy is substantially less than these values, then the postshock temperatures will be very low, resulting in long reaction zone lengths and a decoupled reaction front and shock wave. In order to create a fuel-air mixture in a steady-flow supersonic wind tunnel with the required stagnation conditions, the air must be first heated and then mixed with the fuel after being cooled by expansion through the nozzle. Detonations can be established in wind tunnel test sections using mixtures with lower stagnation enthalpies, but the waves will be unsteady (Bellet and Deshayes 1970) since the Mach number in test section will be lower than the CJ value.

Early results that were interpreted as detonations (Gross and Chinitz 1960) were later shown (Rubins 1960) to be due to combustion upstream of the test section, near the fuel-injection point in the plenum. A similar combustion effect was found in the underexpanded jet studies (Nicholls et al. 1958). Later studies (Rubins and Rhodes 1964, Rhodes et al. 1964) eliminated this effect by relocating the fuel-injection point to a low-temperature portion of the flow. Oblique and normal shocks were produced in a nonuniform mixture of vitiated air (stagnation temperature of $\approx 2000 \mathrm{~K}$ ) and hydrogen flowing with a Mach number of 3.1.

Combustion downstream of the shocks was observed in later tests, and this was properly interpreted as being shock-induced combustion rather than a detonation phenomena. This was due to the very narrow region of fuel-air mixture produced by the mixing of a fuel jet originating from an injector in the nozzle region of the tunnel. The reaction zone lengths in these experiments were comparable to the width of the jet. Under these conditions, little coupling would be expected between shock and the chemical reaction kinetics. Only when the flow behind the shock was confined by a tube or channel was significant coupling observed (Rubins and Cunningham 1965, Strehlow and Rubins 1969). However, no detonation-like structures were ever observed but only shock oscillations associated with choking within 
the duct.

Instead of attempting to create a high-speed, combustible flow, an alternative is to send a high-velocity projectile through a stationary flow. The first experiments of this type were carried out by Ruegg and Dorsey (1962) and later by Behrens et al. (1965), McVey and Toong (1971), Alpert and Toong (1972), and Lehr (1972). These experiments were carried out with different fuel-air and fuel-oxygen mixtures and small-diameter projectiles (10 to $20 \mathrm{~mm}$ diam.). In all cases, it appears that either the projectile was too small or the velocity was too low (less than the CJ value) to obtain detonations. In these situations, the reaction front decoupled from the shock front as shown in Fig. 15a.

Ruegg and Dorsey used stoichiometric hydrogen-air mixtures at $0.1,0.25$ and $0.5 \mathrm{~atm}$. The spherical projectiles $(20 \mathrm{~mm}$ diam. $)$ were launched at various velocities between 1640 and $2665 \mathrm{~m} / \mathrm{s}$. Behrens et al. used $9 \mathrm{~mm}$ projectiles fired at velocities between 1500 to $3000 \mathrm{~m} / \mathrm{s}$ into stoichiometric hydrogen-air mixtures at initial pressures of 0.25 and $0.55 \mathrm{~atm}$. They demonstrated the correlation of the instability frequencies with the induction time in the stagnation region and also observed that most of the shocked gas is unburned due to the decoupling of the shock and reaction fronts that is shown in Fig. 15a. Although some velocities were in excess of the CJ velocity (1920 to $1960 \mathrm{~m} / \mathrm{s})$, the estimated cell widths $(145$ to $27 \mathrm{~mm}$ ) were all substantially larger than the projectile so detonation would not be expected in either sets of experiments.

McVey and Toong fired $12.7 \mathrm{~mm}$ and $6.5 \mathrm{~mm}$ diam. projectiles into lean acetylene-oxygen mixtures at pressures from 50 to 200 Torr. Alpert and Toong examined $12.7 \mathrm{~mm}$ diam. spheres and cylinders fired into stoichiometric hydrogen-oxygen mixtures diluted with argon or nitrogen at pressures between 100 and 532 Torr. Similar conclusions about the cell width and projectile size apply to these experiments and explain the failure to observe a detonation mode of combustion.

Lehr's experiments came the closest to meeting the conditions for creating detonations but the projectile velocity was slightly too low even in the most favorable case. Among other tests, he fired 15 $\mathrm{mm}$ diam. sphere-cylinder projectiles into stoichiometric hydrogenoxygen mixtures with an initial pressure of 186 Torr. Transient detonation waves were initiated for projectile velocities of 2160 and 2705 $\mathrm{m} / \mathrm{s}$ but they were not stabilized on the projectiles since the highest 
velocity used was less than the CJ velocity of $2750 \mathrm{~m} / \mathrm{s}$. The cell width for this mixture is about 4 to $6 \mathrm{~mm}$, less than half the width of the projectile. The characteristic cellular instability structure of a coupled reaction front-shock wave complex can be clearly observed in the schlieren photographs of these events (Lehr 1972). A hypothetical stabilized detonation configuration can be deduced from these tests and is shown in Fig. 15b.

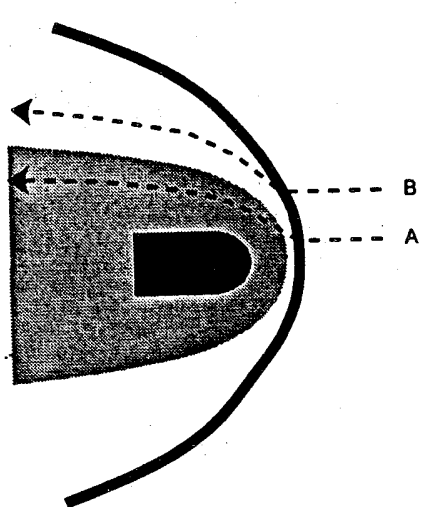

(a)

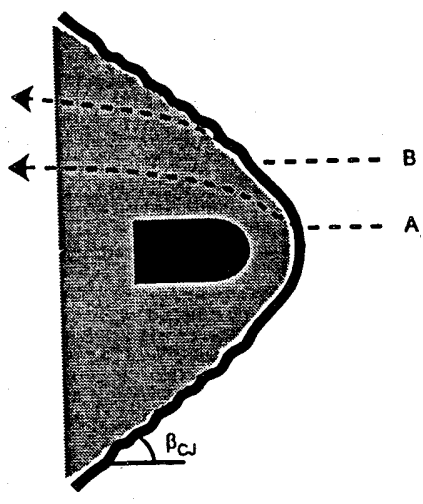

(b)

Figure 15: Combustion waves produced by hypersonic projectiles a) decoupled shock wave and reaction front configuration. b) hypothetical detonation mode configuration.

There have been a number of analyses of the flowfield produced by hypersonic projectiles in a detonable gas. The application of the hydrodynamic model of detonation and deflagration waves to this problem was extensively analyzed by Chernyi (Chernyi 1966, Chernyi and Gilinskii 1970, Gilinskii et al 1966) with a particular emphasis on self-similar flow fields about wedges and cones. Another aspect of this problem examined in these studies (Levin 1968, Chernyi et al. 1970 ) is the phenomenon of wave splitting and reaction quenching illustrated in Fig. 15a. This is particularly relevant to the problem of detonation initiation and stability for propulsion applications. As demonstrated by the experiments, the overdriven waves in the front of the projectile do not usually smoothly decay into the oblique Chapman-Jouguet waves far from the body but rather abruptly split into a shock and a trailing reaction zone that degenerates into a 
contact surface and eventually, can evolve into a flame. The expansion wave generated by the shoulder of the projectile produces the quenching action that is responsible for this effect. Oscillations of the shock and reaction front may occur during the decay processes. These oscillations are apparently precursors of the transverse instability observed for near-CJ detonation waves.

Sichel and Galloway (1967) and Galloway and Sichel (1969) demonstrated that a simple scaling parameter, $u_{\infty} \tau_{\sigma} / R_{b}$ can be used to classify the blunt body flows from a theoretical viewpoint. In this relation, $R_{b}$ is the body radius and $\tau_{\sigma}$ is the characteristic chemical reaction time. For small values of this parameter, the hydrodynamic model of a detonation wave was useful and far from the projectile, the wave is predicted to asymptote to the CJ condition. For larger values, splitting and quenching of the reaction front is to be expected. This parameter is essentially a measure of the rate of quenching relative to the reaction rate. Models (Fickett and Davis 1979, Bdzil and Stewart 1989) of quasi-steady curved detonations also predict the existence of a critical wave curvature $\kappa$. Steady waves cannot exist if the curvature exceeds some critical level $\kappa_{f}$. Recognizing that the maximum value of $\kappa \propto 1 / R_{b}$, we infer that wave splitting would be expected whenever $R_{b}$ is significantly smaller than $1 / \kappa_{f}$. We expect that these considerations could play an important role in determining the characteristics of stabilized curved detonation waves in combustors.

A number of researchers (McVey and Toong 1971, Matsuo and Fujiwara 1993, Wilson and Sussman 1993) have examined the periodic instabilities that occur in these flows. These instabilities are a consequence of the reflection of waves between the shock front, reaction front and the projectile. Convection of these disturbances into the wake of the body produce a striking periodic pattern. These patterns are not to be confused with the cellular instability of detonations. The leading shock waves in these experiments are smooth and free from any transverse instabilities since the reaction front lags far behind the shock in the region far from the projectile.

Oblique detonations have actually been observed in only one type of experiment, the two-layer detonations of Liu et al. 1987, Liu et al. 1988 and Dabora et al. 1991. A channel is divided longitudinally by a rigid partition terminating in a flexible film or diaphragm, Fig. 14d. The upper portion of the channel is filled with the "primary" explosive that has a high detonation velocity and the lower 
portion of the channel is filled with a "secondary" explosive with a lower detonation velocity. A detonation is initiated in the primary layer and propagates in the upper channel toward the film or diaphragm region. When the primary detonation propagates over the film or diaphragm, the high pressure gas behind primary detonation drives the film or diaphragm into the secondary layer with an oblique piston action. This produces a shock wave in the secondary explosive and initiates through a complex system of waves (Oran et al. 1992), an oblique propagating detonation in the secondary explosive. Reflections from the lower boundary of the secondary layer may play a significant role in the initiation process and a Mach stem may be created in the shock or detonation reflection process.

After the transient initiation processes have ceased, the oblique detonation wave angle can be predicted (Liu et al. 1987) by a simple steady flow analysis in the frame of the shock intersection located at the boundary between the primary and secondary layers. In general, a reflected wave (usually an expansion) is produced in the products of the primary explosive in order to match the flow deflection angle and pressure at the contact surface between the products of the primary and secondary explosive. This simple picture is complicated by the presence of an initiation region near the boundary and possibility of an irregular refraction process if the CJ detonation velocity is higher in the secondary explosive than in the primary.

Oblique detonations are not always produced in these experiments. In order to get coupling of the reaction front with the oblique shock, the reaction zone must be small enough compared to the lateral extent of the layer. The initiating shock must also be sufficiently strong to cause initiation without too much delay. The reaction zone length in the primary explosive must also be sufficiently small compared to the channel width so that the expansion wave created by the interaction does not quench the detonation in the primary layer. This is related to the critical tube diameter problem of propagating detonations that was mentioned previously. Failure to initiate the secondary detonation and in some cases, failure of the primary wave itself may result if these conditions are not satisfied.

Oblique detonations were produced using hydrogen-oxygen mixtures with a rich primary mixture and a lean secondary mixture by Liu et al. 1987 and Liu et al. 1988. The lateral extent of each channel was about $16 \mathrm{~mm}$ and the two mixtures were initially separated by a thin cellulose layer in the interaction region. A schlieren photograph 
of the interaction is shown in Fig. 16 from a more recent study by Tonello and Sichel (1993). Note the trailing transverse waves characteristic of cellular instability structure on both the primary and oblique waves. There is also a pronounced initiation shock near the boundary and the reflected waves generated by the interactions are clear. Interaction with a tertiary layer at the bottom of the channel is also visible in this photograph but not germane to the present discussion. The secondary wave angle abruptly increases due to the pressure waves generated by the explosion of the fluid elements in the secondary material close to the primary-secondary contact surface.

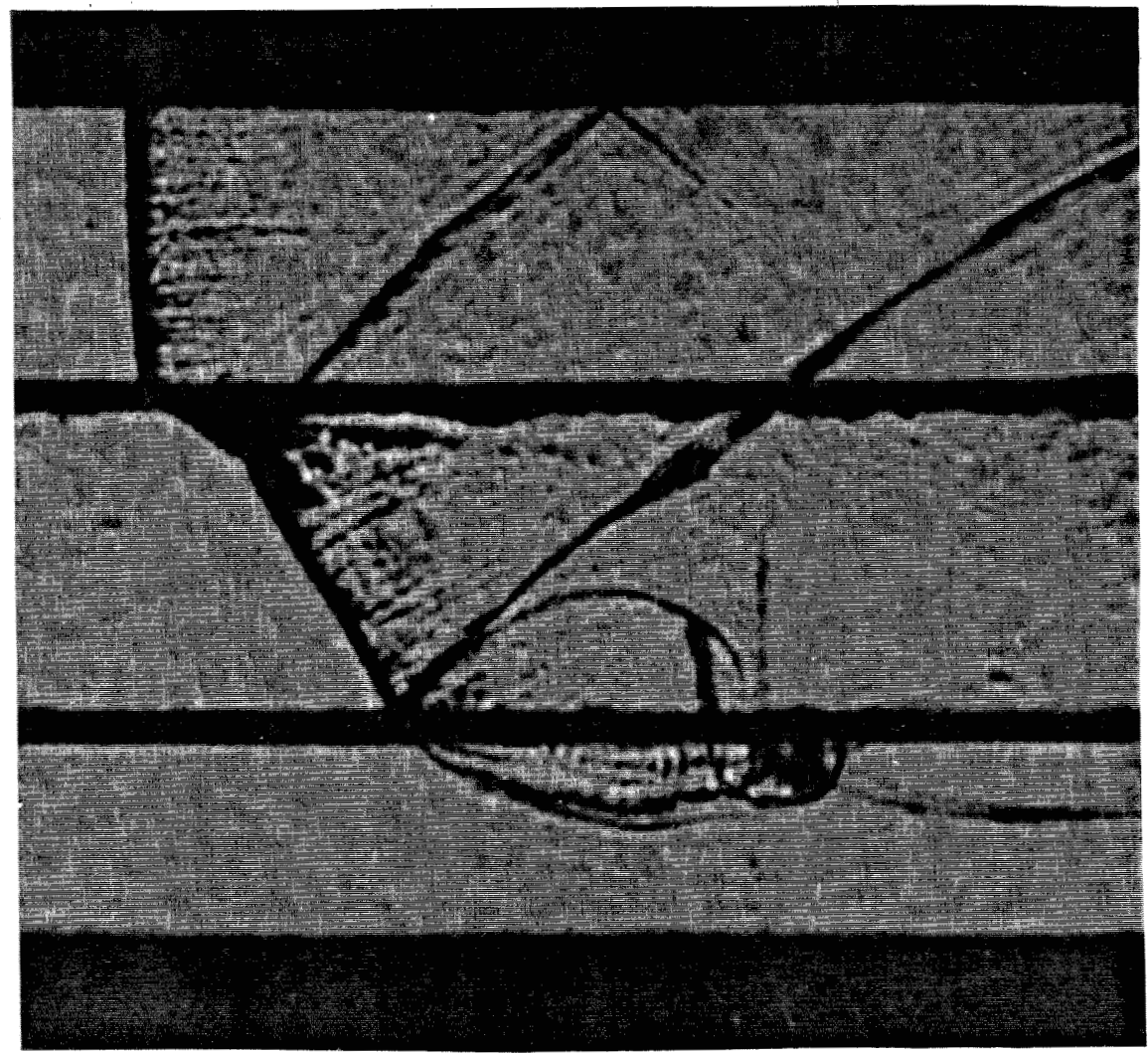

Figure 16: Schlieren photograph of the oblique detonation wave produced by a primary explosive of $2 \mathrm{H}_{2}+1 / 2 \mathrm{O}_{2}$ diffracting into a secondary explosive of $0.57 \mathrm{H}_{2}+1 / 2 \mathrm{O}_{2}$. Both explosives are at an initial pressure of $1 \mathrm{~atm}$ and a temperature of $300 \mathrm{~K}$. From Tonello and Sichel 1993. 
-IəКет К.Iерuоoว

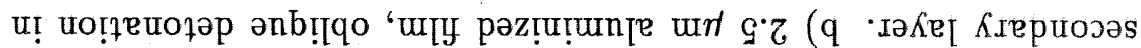

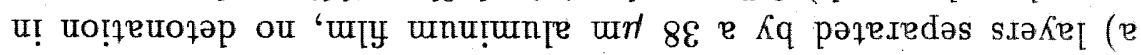

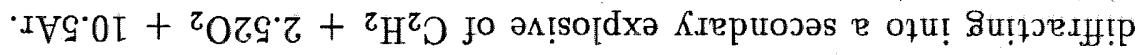

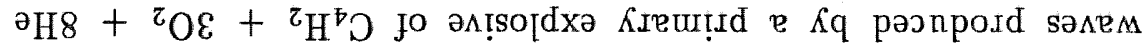

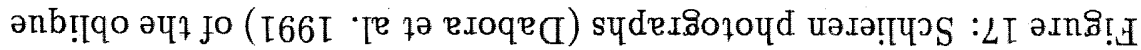

(q)

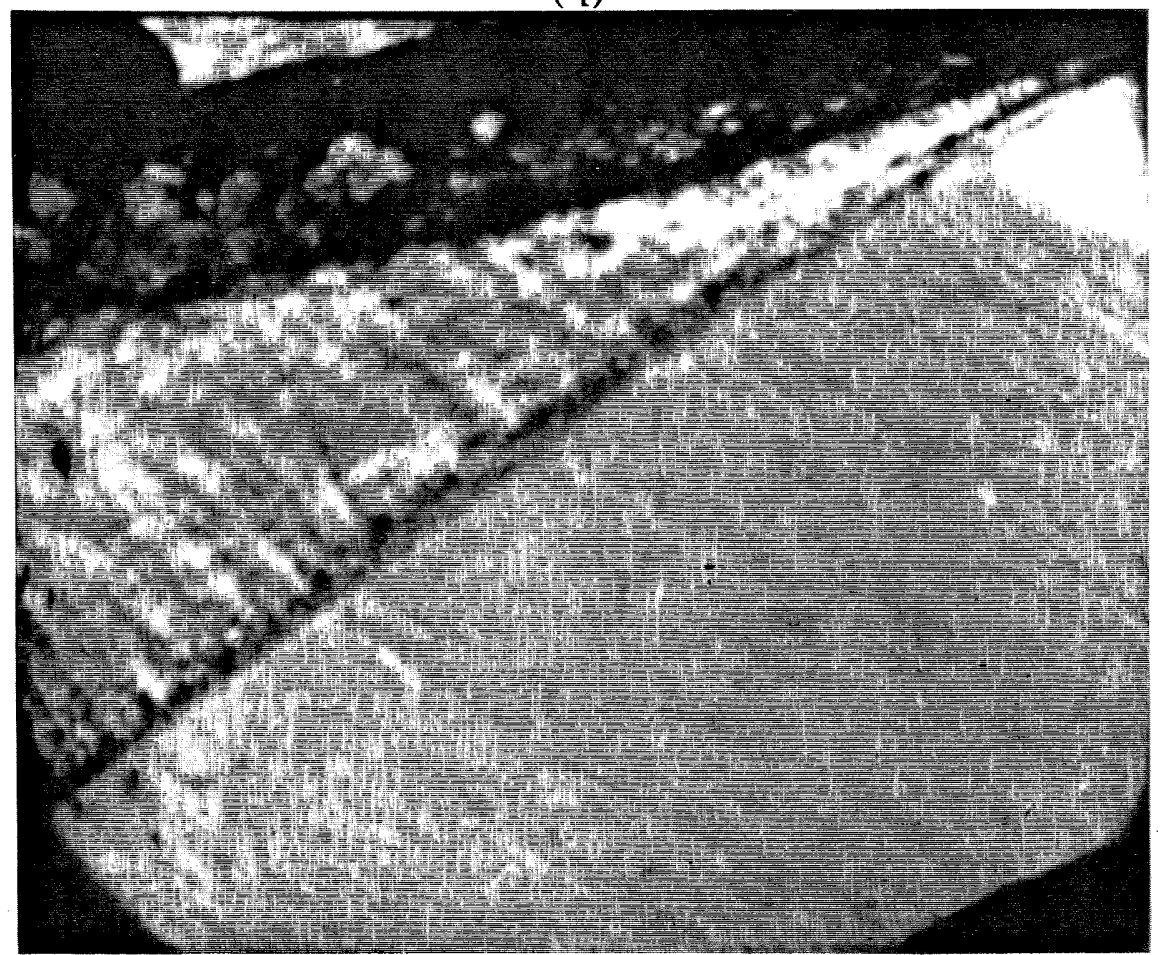

(e)

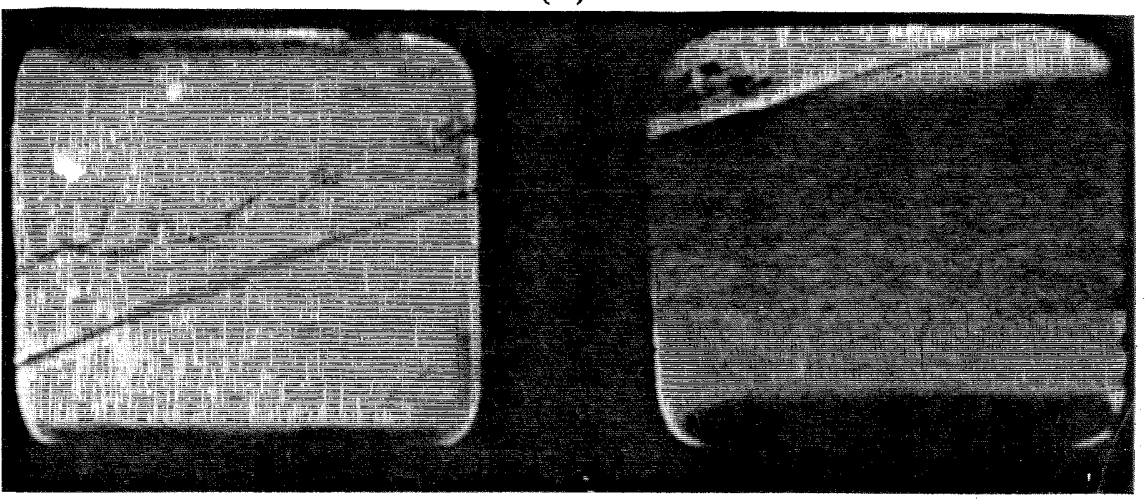


An example in which a failure to obtain an oblique detonation is shown in Fig. 17a. Dabora et al. (1991) used a primary mixture of $\mathrm{C}_{2} \mathrm{H}_{4}+3 \mathrm{O}_{2}+8 \mathrm{He}\left(U_{C J}=3050 \mathrm{~m} / \mathrm{s}\right)$ to drive an $\mathrm{Al}$ foil into a mixture of $\mathrm{C}_{2} \mathrm{H}_{2}+2.5 \mathrm{O}_{2}+10.5 \mathrm{Ar}\left(U_{C J}=1750 \mathrm{~m} / \mathrm{s}\right)$. Each layer is approximately $56 \mathrm{~mm}$ high. Only an oblique shock is observed in the secondary layer with $38 \mu \mathrm{m}$ thick foil. When the foil thickness is reduced to $2.5 \mu \mathrm{m}$ aluminized mylar, an oblique detonation complete with the characteristic instability waves is observed (Fig. 17b). Broda and Dabora (1993) have demonstrated that thick foils slowly accelerate due to their large specific inertia. This produces a weak initial oblique shock that fails to initiate a detonation.

\section{Waves on Wedges, Instabilities and Initiation}

The simplest concept for creating oblique detonations is to just introduce a wedge or ramp into a uniform flow. Chernyi (1966) considered this flow and the axisymmetric analog, flow over a cone, in some detail. Pratt et al. (1991) considered the two-dimensional flow, particularly the situation with a uniform flow downstream. In addition, there have been a number of recent theoretical studies ( $\mathrm{Li}$ et al. 1993, Powers and Stewart 1992, Cambier et al. 1989, Buckmaster and Lee 1990, Buckmaster 1990 ) of the two-dimensional flow over a wedge or a cone.

The possible steady oblique wave configurations can be determined by analyzing the shock polars (Figs. 8 and 9). The results are summarized in Fig. 18a on the velocity-flow deflection plot. First of all, the incoming flow velocity $u_{1}$ must be greater than the CJ velocity $w_{C J}$ in order for a stabilized detonation to exist. Otherwise, an unsteady detonation wave or shock-induced combustion will result. If the velocity is large enough, then there are several regime of stabilized waves, depending on the wedge angle $\theta_{w}$.

A straight wave with uniform flow downstream (Fig. 18b) is possible only if the wedge angle is compatible with the flow deflection angle $\theta$. Referring to the polar plot Fig. 8, this will occur only for $\theta_{C J}<\theta_{w}<\theta_{\max }$, which is a function of the incoming flow velocity as shown in Fig. 18. This is the case considered in most studies. Note this region is rather narrow near the CJ velocity but is quite substantial at higher velocities. However, for large $u_{1}$ and $\theta_{w}$, solutions are obtained (region to the right of dashed line in Fig. 18a) for which the reaction zone is net endothermic, an undesirable situation 
for propulsion purposes.

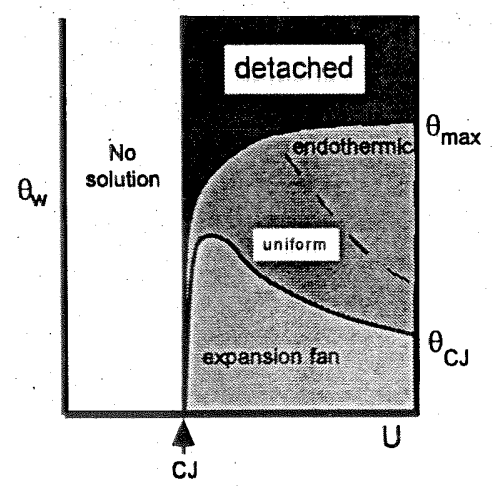

(a)

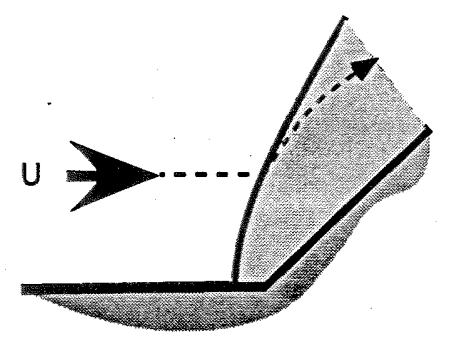

(c)

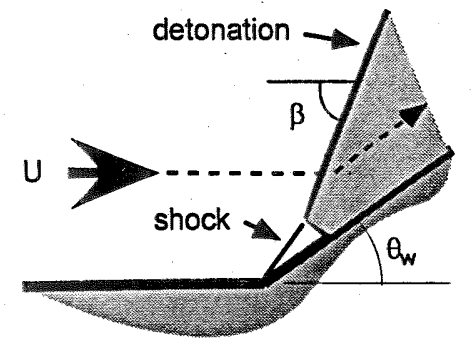

(b)

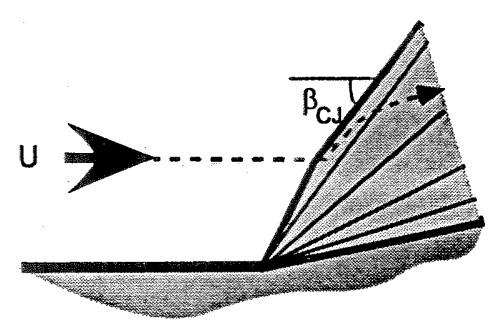

(d)

Figure 18: Regimes of oblique detonation stabilization on wedges. a) possible configurations as determined from a polar analysis. b) transition to uniform downstream flow after a shock initiation transient. c) detached wave over a steep wedge. d) CJ wave followed by an expansion fan.

Solutions with curved waves and nonuniform flow downstream are possible for wedge angles outside this range. For steep wedges, $\theta_{w}>\theta_{\max }$, the wave will be detached (Fig. 18c) just as in ordinary shock waves over blunt bodies. For shallow wedges, $\theta_{w}<\theta_{C J}$, the detonation wave will be followed by an expansion fan (Fig. 18d) and the wave angle will be given by the CJ value. This is the steady ana$\log$ of the self-sustained detonation. The conical version of this flow was examined by Chernyi (1966) and more recently in the context of 
propulsion by Carrier et al. (1992).

The polar discussion is based on the notion of a thin wave with negligible reaction zone structure effects. Reaction zone effects will be of two sorts. First, the detonation wave will possibly be unstable. Second, there will be some sort of initiation transient.

The usual transverse wave instability of propagating detonations is certainly possible and has been observed on oblique detonations in the two-layer experiments discussed previously. Based on experience with propagating waves, we speculate that unless the cell width becomes comparable to the combustor transverse dimension, this instability will not cause the detonation wave to fail. The extent of the instability depends crucially on the amount of overdrive, $w / w_{C J}$. Experiments with overdriven detonations (Meltzer et al. 1993) indicate that the cell width decreases by a factor of 10 with an overdrive ratio of 1.35 and the instability is completely suppressed at an overdrive ratio of 1.4. Recent experiments with the two-layer configuration (Broda and Dabora 1993) indicate a similar decrease in cell width with overdrive. Although a slight overdrive might be beneficial in a propulsion system, a significant loss in performance for a propulsion system may be incurred by operating at too high an overdrive. The total pressure loss across the wave increases rapidly with overdrive and therefore the net thrust will decrease.

The initiation transient is observed as a precursor shock (see Figs. 16 and 17) in the two-layer experiments discussed previously. A similar precursor would be expected in front of the wedge, as indicated in Fig. 18b. Structures of this sort are visible in the computations of $\mathrm{Li}$ et al. (1993). Inspection of the polar curves (Figs. 8 and 9) reveals that a nonreactive shock with a given flow deflection angle is much weaker than the leading shock portion of a detonation with a given flow deflection angle in the products. In effect, the exothermic reaction increases the effective wedge angle. This has rather severe implications for starting and maintaining oblique detonations. Since the key reaction rates depend strongly on temperature, a weak precursor shock will result in a much longer reaction zone (up to $10^{5}$ times larger than for near-CJ waves!) than behind the leading shock of a steady oblique detonation wave on the same wedge.

Consequences of this include postshock reaction zones that are so long in comparison to the combustor width that no detonation is ever established. Another possibility is that the detonation wave is catastrophically unstable to disturbances that decouple the leading 
shock from the energy-releasing portion of the reaction zone. Such decoupling occurs in the projectile flows (Fig. 15a) and is another manifestation of the sensitivity of the reaction zone length to postshock temperature. This type of instability effect for wedge flows has been analyzed by Buckmaster (1990) and a polar analysis of the decoupled configuration was given by Buckmaster and Lee (1990). Clearly, some special measures will be needed to initiate the detonation wave in this type of flow. External initiation or programmed excursions in the ramp angle may be required in order to successfully start an oblique detonation. These considerations may also apply to certain types of numerical simulations.

\section{Conclusions}

There are many unresolved issues related to using detonation waves as the basis combustion process in a hypersonic propulsion system. A few of these have been examined in this paper. The theoretical ideas that have been presented are rather rudimentary and focus on idealized models of detonation wave structure. It is apparent that resolution of many issues requires investigation into unsteady and multidimensional flows. Sophisticated experimentation and numerical simulation will certainly be required to make further progress. However, the simple considerations of this paper indicate that there are some key parameters and physical phenomena that should be carefully considered in future studies.

The role of the reaction zone structure and the spontaneous instability of detonation waves appear to be crucial factors that complicate any study, experimental or numerical. The reaction zone length or detonation cell width provide key scaling parameters that should be considered in the choice of experimental setup or computational domain. The characteristic dimensions of the combustor should be large enough to accommodate at least several cell widths $\lambda$ or several hundred times the ZND reaction zone length $\Delta$. In addition, the upstream flow velocity and stagnation enthalpy must be sufficiently high in order to initiate and maintain a stabilized detonation. Flow disturbances must be minimized in order not to cause decoupling and catastrophic failure. Finally, even with carefully designed experiments, there may be more than one steady state depending on the nature of the initiation process. 


\section{Acknowledgements}

E. K. Dabora and M. Sichel generously provided photographs and shared the results of their oblique detonation wave experiments with me. I would like to thank P. M. Rubins for providing his unique perspective and papers about the early research on shock-induced combustion. J. H. S. Lee pointed out many connections and provided references to related work. B. McBride graciously provided an early version of the NASA high-temperature thermodynamic property fits.

Several of my students, J. Meltzer, R. Akbar, and A. I. Sabet, have contributed through their studies on detonation-related problems. In particular, A. I. Sabet did many of the computations on oblique wave polars and reaction zone structures. Most of this work and writing was carried out at Rensselaer Polytechnic Institute in Troy, NY. A. Kapila, M. Y. Hussiani, J. Quirk, and T. Jackson provided encouragement and a forum for these ideas at ICASE.

\section{References}

Alpert, R. L. and Toong, T. Y., 1972. "Periodicity in Exothermic Hypersonic Flows about Blunt Projectiles," Astronautica Acta 17, pp. 539-560.

Atamanchuk, T., and Sislian, J., 1990. "On-and-Off Design Performance Analysis of Hypersonic Detonation Wave Ramjets," 26th Joint Propulsion Conference, AIAA-90-2473.

Atamanchuk, T., and Sislian, J., 1991. "Hypersonic Detonation Wave Powered Lifting-Propulsive Bodies," 3rd International Aerospace Planes Conference, AIAA-91-5010.

Bach, G. G., Knystautas, R., and Lee J. H., 1969. " Direct initiation of spherical detonation in gaseous explosives," 12th Symp. Comb., The Combustion Institute, Pittsburgh, PA, pp. 853864 .

Bdzil, J. B., and Stewart, D. S., 1989. "Modeling Two-Dimensional detonations with detonation shock dynamics," Phys. Fluids A1(7), pp. 1261-1267.

Behrens, H., Struth, W., and Wecken, F., 1965. "Studies of hypervelocity firings into mixtures of hydrogen with air or oxygen," 
10th Symp. (Intl.) Comb., The Combustion Institute, Pittsburgh, PA, pp. 245-252.

Bellet, J. C., and Deshayes, G., 1970. "Structure and Propagation of Detonations in Gaseous Mixtures in Supersonic Flow," Astronautica Acta 15, pp. 465-469.

Bourlioux, A. and Madja, A. J., 1992. "Theoretical and Numerical Structure for Unstable Two-Dimensional Detonation," Combust. Flame 90, pp. 211-229.

Broda, J.-C., and Dabora, E. K., 1993. "Recent Experimental Results on Oblique Detonation Waves," Presented at the 14th ICDERS Symposium, August 1993, Coimbra, Portugal.

Buckmaster, J. D. 1990. "The Structural Stability of an Oblique Detonation Wave," Combust. Sci. Tech. 72, 283-296.

Buckmaster, J. D., and Lee, C. J., 1990. "Flow Refraction by an Uncoupled Shock and Reaction Front," AIAA J. 28, pp. 13101312.

Cambier, J.-L., Adelman, H. G., Menees, G. P., 1989. "Numerical Simulations of Oblique Detonations in Supersonic Combustion Chambers", J. Propulsion Power, 5(4), pp. 482-491.

Carrier, G., Fendell, F., McGregor, R., Cook, S., and Vazirani, M., 1992. "Laser-Initiated Conical Detonation Wave for Supersonic Combustion," J. Propulsion Power 8, pp. 472-480.

Chase, M. W., Jr., Davies, C. A., Downey, J. R., Jr., Frurip, D. J., McDonald, R. A., and Syverud, A. N., 1985. JANAF Thermochemical Tables, Third Edition. J. Phys. Chem. Ref. Data 14, Supplement No. 1.

Chernyi, G. G., 1966. "Self-similar Problems of Combustible Gas Mixture Flow Past Bodies," Mekhanika Zhidkosti i Gaza (translated in english as Fluid Dynamics) 1(6), pp. 10-24.

Chernyi, G. G., and Gilinskii, S. M., 1970. "High-Velocity Motion of Solid Bodies in Combustible Gas Mixtures," Astronautica Acta 15, pp. 539-545. 
Chernyi, G. G., Korobeinikov, V. P., Levin, V. A., and Medvedev, S. A., 1970. "One-Dimensional Unsteady Motion of Combustible Gas Mixtures Associated with Detonation Waves," Astronautica Acta 15, pp. 259-256.

Dabora, E. K., Desbordes, D., Guerraud, C., Wagner, H. Gg., 1991. "Oblique Detonations at Hypersonic Velocities" Prog. Aero. Astro. 133, 187-204.

Dunlap, R., Brehm, R. L., and Nicholls, J. A., 1958. "A Preliminary Study of the Application of Steady State Detonative Combustion to a Reaction Engine," Jet Propulsion 28, pp. 451-456.

Eidelman, S., Grossman, W., and Lottati, I. 1991 "Review of Propulsion Applications and Numerical Simulation of the Pulsed Detonation Engine Concept," J. Propulsion Power 7, pp. 857-865.

Fickett, W. and Davis, W. C., 1979. Detonation, University of California Press, Berkeley, California.

Galloway, A. J., and Sichel, M., 1969. "Hypersonic Blunt Body Flow of $\mathrm{H}_{2}-\mathrm{O}_{2}$ Mixtures," Astronautica Acta 15, pp. 89-105.

Gilinskii, S. M., Zapryanov, Z. D., and Chernyi, G. G., 1966. "Supersonic flow of a Combustible Gas Mixture Past a Sphere," Mekhanika Zhidkosti i Gaza (translated in english as Fluid Dynamics) 1(5), pp. 8-13.

Gordon, S. and McBride, B. J., 1976. "Computer Program for the Calculation of Complex Chemical Equilibrium Compositions, Rocket Performance, Incident and Reflected Shocks and Chapman-Jouguet Detonations." NASA SP-273.

Gross, R. A. and Chinitz, W., 1960. "A Study of Supersonic Combustion," J. Aero. Science 27(7), pp. 517-524.

Harradine, D. M., Lyman, J. L., Oldenborg, R. C., Schott, G. L., Watanabe, H. H., 1990. "Hydrogen/Air Combustion Calculations: The Chemical Basis of Efficiency in Hypersonic Flows" AIAA J. 28, pp. 1740-1744.

Hindmarsh, A. C., 1983. "ODEPACK, A Systemized Collection of ODE Solvers," IMACS Trans. on Scientific Computation 1, pp. 55-64. 
Jackson, T. L., Kapila, A. K., and Hussaini, M. Y., 1990. "Convection of a pattern of vorticity through a reacting shock wave," Phys. Fluids A2 1260-1268.

Kee, R. J., Rupley, F. M., and Miller, J. A., 1989. "CHEMKIN II: A FORTRAN Chemical Kinetics Package for the Analysis of Gas-Phase Chemical Kinetics," Sandia National Laboratories Report SAND90-8009.

Lasseigne, D. G., and Hussaini, M. Y., 1993. "Interaction of disturbances with an oblique detonation wave attached to a wedge," Phys. Fluids A5, 1047-1058.

Lee, J. H., 1984. "Dynamic Parameters of Gaseous Detonations," Ann. Rev. of Fluid Mech. 16, pp. 311-336.

Lee, H., and Stewart, D. S., 1990. "Calculations of Linear Detonation Instability," J. Fluid Mech. 216, pp. 103-132.

Lehr, H. F., 1972. "Experiments on Shock-Induced Combustion," Astronautica Acta 17, pp. 589-597.

Levin, V. A., 1968. "Transition of a Plane Overdriven Detonation Wave to the Chapman Jouguet Regime" Mekhanika Zhidkosti $i$ Gaza (translated in english as Fluid Dynamics) 3(2), pp. 50-55.

Liepmann, H. W. and Roshko, A., 1957. Elements of Gasdynamics, John Wiley, New York.

Li, C., Kailasanath, K., and Oran, E. S., 1993. "Structure of Reaction Waves Behind Oblique Shocks," Prog. Astro. Aero. 153, pp. 231-240.

Liu, J. C., Liou, J. J., Sichel, M., Kaufmann, C. W., and Nicholls, J. A., 1987." "Diffraction and Transmission of a Detonation into a Bounding Explosive Layer," 21st Symposium (International) on Combustion, The Combustion Institute, Pittsburgh, PA, pp. 1639-1647.

Liu, J. C., Sichel, M., and Kaufmann, C. W., 1988. "The lateral interaction of detonating and detonable gaseous mixtures," Prog. Astro. Aero. 114, AIAA, pp. 264-283. 
Lutz, A. E., Kee, R. J., Miller, J. A., Dwyer, H. A., and Oppenheim, A. K., 1988. "Dynamic Effects of Autoignition Centers for Hydrogen and $\mathrm{C}_{1,2}-\mathrm{Hydrocarbon} \mathrm{Fuels,"} \mathrm{22nd} \mathrm{Symp.} \mathrm{(Int.)}$ on Combust., pp. 1683-1693, The Combustion Institute, Pittsburgh, PA.

Matsuo, A. and Fujiwara, T., 1993. "Numerical Prediction of Mechanism on Oscillatory Instabilities in Shock-Induced Combustion," Progress in Astonautics and Aeronautics 154, AIAA, Washington, DC, pp. 516-531.

McVey, J. B. and Toong, T. Y ., 1971. "Mechanism of Instabilities of Exothermic Hypersonic Blunt-Body Flows," Combust. Sci. Tech. 3, pp. 63-76.

Meltzer, J., Shepherd, J. E., Akbar, R., Sabet, A., 1993. "Mach Reflection of Detonation Waves," Progress in Astronautics and :Aeronautics 153, AIAA, Washington, DC, pp. 78-94.

Murthy, S. N. B., and Curran, E. T., Editors. 1991 High-Speed Flight Propulsion Systems in Progress in Astronautics and Aeronautics 137, AIAA, Washington, DC.

Nicholls, J. A., Dabora, E. K., and Gealer, R. L., 1959. "Studies in Connection with Stabilized Gaseous Detonation Waves," 7th Symp. (Intl.) Comb., Butterworths, London, pp. 766-772.

Nicholls, J. A., 1963. "Standing Detonation Waves," gth Symp. (Intl.) Comb., Academic Press, pp. 488-498.

Oran, E. S., Jones, D. A., and Sichel, M., 1992. "Numerical simulations of detonation transmission," Proc. Roy. Soc. Lond. A 436, pp. 267-297.

Ostrander, M. J., Hyde, J. C., Young, M. F., Kissinger, R. D., and Pratt, D. T., 1987. "Standing Oblique Detonation Wave Engine Performance," AIAA paper 87-2002.

Powers, J. M., and Stewart, D. S., 1992. "Approximate Solutions for Oblique Detonations in the Hypersonic Limit," AIAA J. 30, pp. 726-736. 
Pratt, D. T., Humphrey, J. W., Glenn, D. E, 1991. "Morphology of Standing Oblique Detonation Waves," J. Propulsion Power 7(5), pp. 837-845.

Reynolds, W. C., 1986. "The Element Potential Method for Chemical Equilibrium Analysis: Implementation in the Interactive Program STANJAN; Version 3", Department of Mechanical Engineering, Stanford University.

Rhodes, Jr., R. P., Rubins, P. M., and Chriss, D. E., 1964. "Effect of Heat Release on Flow Parameters in Shock Induced Combustion," SAE Transactions 72, pp. 87-95.

Rubins, P. M., 1960. "Installation and Calibration of a Supersonic Combustion Tunnel," AEDC-TN-60-162.

Rubins, P. M., and Rhodes, Jr., R. P., 1964. "Shock-Induced Combustion with Oblique Shocks: Comparison of Experiment and Kinetic Calculations," AIAA J. 1(12), pp. 2778-2784.

Rubins, P. M., and Cunningham, T. H. M., 1965. "Shock-Induced Supersonic Combustion in a Constant-Area Duct," J. Spacecraft Rockets 2(2), pp. 199-205.

Ruegg, F. W. and Dorsey, W. W., 1962. "A Missile Technique for the Study of Detonation Waves," J. Research of the NBS 66C(1), pp. 51-58.

Sabet, A. I., 1990."Investigation of Equilibrium and Chemical Kinetic Behaviour of Detonation Waves in Hydrogen-Air Mixtures and an Evaluation of the Oblique Detonation Wave as the Combustor for a Scramjet", Masters Thesis, Rensselaer Polytechnic Institute, Troy, New York.

Sangiovanni, J. J., Barber, T. J., and Syed, S. A., 1993. "Role of Hydrogen/Air Chemistry in Nozzle Performance for a Hypersonic Propulsion System," J.Propulsion Power 9, 134-138.

Sargent, W. H., and Gross, R. A., 1960 "Detonation Wave Hypersonic Ramjet," ARS J. 30, pp. 543-549.

Shepherd, J. E., 1986. "Chemical Kinetics of Hydrogen-Air-Diluent Detonations," Prog. Astro. Aero. 106, pp. 263-293. 
Sichel, M., and Galloway, A. J., 1967. "Regimes of Exothermic Blunt Body Flow," Astronautica Acta 13, pp. 137-145.

Strehlow, R. A., 1984. Combustion Fundamentals, McGrawHill, NY.

Strehlow, R. A. and Rubins, P. M., 1969. "Experimental and Analytical Study of $\mathrm{H}_{2}$-Air Reaction Kinetics Using a Standing Normal Shock Wave," AIAA J. 7, pp. 1335-1344.

Thompson, P. A., 1972. Compressible Fluid Dynamics, McGrawHill, NY.

Tonello, N. A. and Sichel, M., 1993. Personal communication. See also Tonello, N. A., Sichel, M., and Kaufmann, C. W., "Mechanisms of Detonation Transmission in Layered $\mathrm{H}_{2}-\mathrm{O}_{2}$ Mixtures" Presented at the 14th ICDERS Symposium, August 1993, Coimbra, Portugal.

Voytsekhovskiy, B. V., Mitrofanov, V. V., and Topchiyan, M. Ye., 1964. "Struktura Fronta Detonatskii v Gazakh," Novosibirsk. Available in english as "The structure of a Detonation Front in Gases," Air Force Translation FTD-MT-64-527.

Westbrook, C. K. and Urtiew, P. A., 1982. "Chemical Kinetic Prediction of Critical Parameters in Gaseous Detonations," 19th Symposium (Intl.) on Combustion, pp. 615-623, The Combustion Institute, Pittsburgh, PA.

Wilson, G. J., and Sussman, M. A., 1993. "Computation of Unsteady Shock-Induced Combustion Using Logarithmic Species Conservation Equations," AIAA J. 31, pp. 294-301.

Zeldovich, Ia. B., and Kompaneets, A. S., 1960. Theory of Detonation, Academic Press, NY. 